\title{
Coherent resonance stop bands in alternating gradient beam transport
}

\author{
K. Ito, H. Okamoto, ${ }^{*}$ Y. Tokashiki, and K. Fukushima ${ }^{\dagger}$ \\ Graduate School of Advanced Sciences of Matter, Hiroshima University, \\ 1-3-1 Kagamiyama, Higashi-Hiroshima 739-8530, Japan
}

(Received 23 February 2017; published 22 June 2017)

\begin{abstract}
An extensive experimental study is performed to confirm fundamental resonance bands of an intense hadron beam propagating through an alternating gradient linear transport channel. The present work focuses on the most common lattice geometry called "FODO" or "doublet" that consists of two quadrupoles of opposite polarities. The tabletop ion-trap system "S-POD" (Simulator of Particle Orbit Dynamics) developed at Hiroshima University is employed to clarify the parameter-dependence of coherent beam instability. S-POD can provide a non-neutral plasma physically equivalent to a chargedparticle beam in a periodic focusing potential. In contrast with conventional experimental approaches relying on large-scale machines, it is straightforward in S-POD to control the doublet geometry characterized by the quadrupole filling factor and drift-space ratio. We verify that the resonance feature does not essentially change depending on these geometric factors. A few clear stop bands of low-order resonances always appear in the same pattern as previously found with the sinusoidal focusing model. All stop bands become widened and shift to the higher-tune side as the beam density is increased. In the spacecharge-dominated regime, the most dangerous stop band is located at the bare betatron phase advance slightly above 90 degrees. Experimental data from S-POD suggest that this severe resonance is driven mainly by the linear self-field potential rather than by nonlinear external imperfections and, therefore, unavoidable at high beam density. The instability of the third-order coherent mode generates relatively weak but noticeable stop bands near the phase advances of 60 and 120 degrees. The latter sextupole stop band is considerably enhanced by lattice imperfections. In a strongly asymmetric focusing channel, extra attention may have to be paid to some coupling resonance lines induced by the Coulomb potential. Our interpretations of experimental data are supported by theoretical predictions and systematic multiparticle simulations.
\end{abstract}

DOI: 10.1103/PhysRevAccelBeams.20.064201

\section{INTRODUCTION}

Modern particle accelerators have relied, almost without exception, on the principle of strong focusing [1,2]. Discrete focusing elements, generally quadrupole magnets, are carefully aligned along the design beam orbit to confine a large number of charged particles within tiny transverse space [3]. Each particle receives linear and weak nonlinear forces periodically every turn when the beam orbit is closed. Even in a linear machine, the beam is often focused by an array of identical alternating gradient (AG) quadrupole cells, which makes the external driving potential approximately periodic. This periodic nature of strong focusing systems inevitably gives rise to resonant beam

\footnotetext{
Corresponding author. okamoto@sci.hiroshima-u.ac.jp

Present address: Facility for Rare Isotope Beams, Michigan State University, East Lansing, MI 48824, USA.

Published by the American Physical Society under the terms of the Creative Commons Attribution 4.0 International license. Further distribution of this work must maintain attribution to the author(s) and the published article's title, journal citation, and DOI.
}

instability under a specific condition that depends on the lattice configuration, in other words, the arrangement of quadrupole magnets within a focusing period.

The transverse motion of a single particle traveling in an AG focusing system has been well understood since Courant and Snyder established a most seminal theory over a half century ago [1]. Many papers and standard textbooks published afterwards have followed their theoretical framework. The traditional theory concludes that the so-called betatron motion becomes unstable when the horizontal and vertical bare tunes $\left(\nu_{0 x}, \nu_{0 y}\right)$ satisfy the relation $k \nu_{0 x} \pm \ell \nu_{0 y}=$ integer, where $k$ and $\ell$ are zero or positive integers. The driving potential of this resonance is proportional to $x^{k} y^{\ell}$ with $(x, y)$ being the transverse spatial coordinates. In reality, however, any beams in accelerators contain not one but many particles interacting each other via Coulomb self-fields $[3,4]$. The motion of each individual particle is influenced by those of other particles especially when the beam is dense. The simple single-particle picture no longer applies in such a case. Since the Coulomb interaction has a long range, the beam as a whole exhibits a complex collective behavior responding to the external AG field. The collective motion can be expressed as the 
superposition of various coherent modes whose oscillation frequencies depend on the mode number and beam density. The whole beam becomes resonantly unstable, or more correctly, a certain mode blows up when the frequency of that mode fulfills a specific relation with the frequency of a Fourier harmonic in the periodic driving force.

A systematic experimental study of intense beam stability is very difficult to perform in practice due to the poor controllability of basic parameters. In particular, the AG lattice structure of any accelerator is generally inflexible once constructed. We face severe limitations in exploring how the beam stability depends on the arrangement and dimension of quadrupole magnets. Previous experiments on the resonant instability of space-charge-dominated beams have actually relied on a particular machine with a particular lattice configuration [5-11]. To overcome this practical limitation, we here employ the novel tabletop apparatus called "S-POD" (Simulator of Particle Orbit Dynamics) [12-19]. This experiment is based on an isomorphism between the equations of non-neutral plasma motion in a linear Paul trap (LPT) and the equations of intense beam motion in a strong focusing channel [20]. As explained later, these two many-body systems are governed by very similar Hamiltonians, which guarantees that what happens in one system also happens in the other. We make use of the compact LPT-based facility to approximately reproduce the collective motion of an intense hadron beam in a local tabletop environment.

The main purpose of the present work is the experimental demonstration of fundamental resonance instability bands of an intense hadron beam propagating in the "FODO" (or "quadrupole doublet") lattice with arbitrary geometric factors. In addition to the lattice details, the betatron tunes and beam density are also varied over a very wide range to offer definitive information of beam stability in the most typical AG transport line. The paper is organized as follows. In Sec. II, we first summarize some theoretical expectations regarding the space-charge issue of our interest. Numerical simulation results are given to confirm the existence of a few low-order resonance bands with and without external nonlinear driving forces. After describing in Sec. III how S-POD works, we proceed to experimental observations. In our past experiments with S-POD [14-19], the sinusoidal focusing model was often adopted to approximate the AG focusing effect from the FODO lattice. This simplification is experimentally validated in Sec. IV, and then, we symmetrically modify the lattice geometry to check if any remarkable change is induced in the stop-band distribution. A wide range of asymmetric modification of the focusing (F) and defocusing (D) elements in the FODO lattice is also implemented to survey the transverse tune space. Such a comprehensive experimental study of high-intensity beam transport has never been done elsewhere before. Finally, concluding remarks are made in Sec. V.

\section{THEORETICAL BACKGROUND}

\section{A. Self-field-driven and external-field-driven resonances}

The transverse dynamics of an intense beam focused by quadrupole magnets obeys the Hamiltonian

$$
H_{0}=\frac{p_{x}^{2}+p_{y}^{2}}{2}+\frac{1}{2} K_{\mathrm{QM}}(s)\left(x^{2}-y^{2}\right)+I_{\mathrm{b}} \phi,
$$

where the path length $s$ along the beam transport line, instead of time $t$, has been chosen as the independent variable, the function $K_{\mathrm{QM}}(s)$ is determined by the $\mathrm{AG}$ lattice structure, $I_{\mathrm{b}}$ is a constant parameter that depends on the beam energy, and $\phi$ is the collective Coulomb potential. In many cases, $K_{\mathrm{QM}}(s)$ is a periodic function; namely, $K_{\mathrm{QM}}(s)=K_{\mathrm{QM}}(s+L)$ with $L$ being the length of an AG focusing cell. In this paper, we consider the most standard lattice configuration illustrated in Fig. 1. This waveform has been referred to as "FODO" or "doublet" in past publications. The cell structure can be characterized by the quadrupole filling factor $\xi$ and the drift-space ratio $\zeta$ defined by $\xi=\left(L_{\mathrm{F}}+L_{\mathrm{D}}\right) / L$ and $\zeta=d_{1} / d_{2}$. In the following, we assume $d_{1} \leq d_{2}$ without loss of generality so that $0 \leq \zeta \leq 1$. The absolute heights of the focusing and defocusing pulses are always set equal. The lattice configuration is then symmetric whenever $L_{\mathrm{F}}=L_{\mathrm{D}}$.

Self-consistency is especially important to draw reliable conclusions in the study of space-charge effects. Provided that Coulomb collisions among individual particles are negligible [21], the time evolution of the phase-space distribution function $f\left(x, y, p_{x}, p_{y} ; s\right)$ is governed by the Vlasov equation

$$
\frac{\partial f}{\partial s}+\left[f, H_{0}\right]=0,
$$

where [,] stands for the Poisson bracket. The Coulomb potential $\phi$ in the Hamiltonian $H_{0}$ is a solution to the Poisson equation

$$
\nabla^{2} \phi=-\frac{q}{\varepsilon_{0}} \iint f d p_{x} d p_{y},
$$

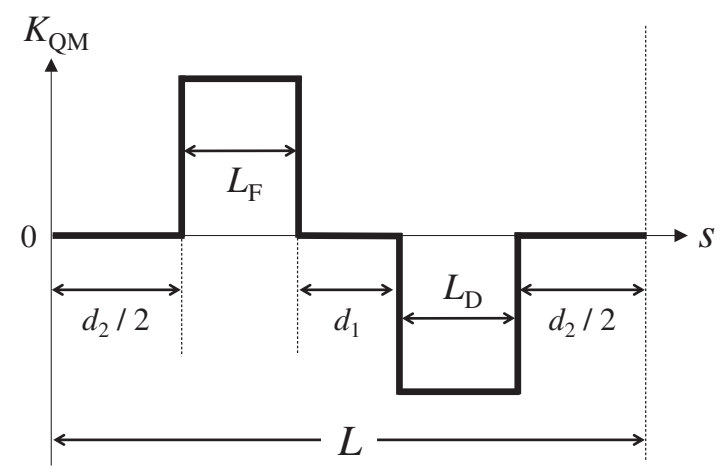

FIG. 1. Quadrupole doublet (FODO) lattice. 
where $q$ is the particle's charge state and $\varepsilon_{0}$ is the vacuum permittivity. To be self-consistent, we must simultaneously solve the Vlasov equation (2) and the Poisson equation (3) together with the Hamiltonian (1) [22-25]. The analytic treatment of this closed set of equations is obviously very difficult if we take the AG lattice design explicitly into account. Almost all theoretical approaches to space-charge issues are thus purely numerical (i.e., computer simulations) or analytic but dependent on the uniform focusing model (i.e., the smooth approximation) [4,26]. As an alternative basis for space-charge studies, the root-meansquared (rms) envelope equations much easier to handle are available [27-31], but they can only describe the linear collective modes that possess elliptical symmetry in real space.

Although the external driving force is completely linear in the Hamiltonian dynamical system of Eq. (1), the nonlinear nature of the Coulomb potential can excite resonant instabilities of higher orders. Considerable theoretical effort was made over thirty years ago by many researchers to figure out the condition of such self-fielddriven instabilities in high-intensity linear transport channels [4]. For instance, Hofmann et al. employed the Kapchinsky-Vladimirsky (KV) model to derive a set of ordinary differential equations from the linearized VlasovPoisson equations and numerically integrated them [24]. It was concluded that the space-charge-induced transverse instability of the $m$ th order could be avoided in a symmetric beam transport line if $\sigma_{0} \leq \pi / m$ where $\sigma_{0}$ denotes the bare betatron phase advance per unit focusing cell. This design criterion was soon confirmed through careful multiparticle simulations by Struckmeier et al [28,32]. Later, Okamoto and Yokoya constructed a fully analytic Vlasov theory in which an arbitrary lattice configuration can be incorporated explicitly [25]. Their theory predicts that the primary parametric resonance of the $m$ th order occurs under the condition

$$
\Omega_{m} \approx \frac{n}{2},
$$

where $\Omega_{m}$ represents the tune of the $m$ th-order collective oscillation mode per lattice period, and $n$ is an integer $[33,34]$. Using the betatron phase advance, Eq. (4) can approximately be rewritten as

$$
m\left(\sigma_{0}-C_{m} \Delta \bar{\sigma}\right) \approx n \pi,
$$

where $\Delta \bar{\sigma}$ is the average phase shift induced by the Coulomb repulsion among particles, and $C_{m}$ is a positive constant less than unity and depending on the mode number $m$. It should be noted that $\Delta \bar{\sigma}$ does not stand for the so-called incoherent tune spread. Denoting the rms tune depression to be $\eta$, we have $\Delta \bar{\sigma}=(1-\eta) \sigma_{0}$ that is almost independent of the phase-space distribution function [27]. A practically important implication of Eq. (5) is that the shift of a collective instability band is somewhat smaller than $\Delta \bar{\sigma}$ because $C_{m}<1$ for all $m$ numbers.

The mathematical description in Ref. [25] clarifies the parameter dependence of the instability and thus gives us useful insight into underlying physics even if it is based on a one-dimensional (1D) model. From Eq. (5), we expect the $m$ th-order resonance to take place around the operating phase advance $\sigma_{0} \approx n \pi / m+C_{m} \Delta \bar{\sigma}$. The 1D Vlasov theory also indicates that the half width of the corresponding resonance band does not exceed the coherent phase shift $C_{m} \Delta \bar{\sigma}$. We can, therefore, eliminate the instability of the $m$ th-order mode by setting $\sigma_{0}$ in the range $\sigma_{0} \leq \pi / m$, which is consistent with the previous numerical finding. Apart from weak coupling resonances, the simple criterion in Eq. (5) works remarkably well to explain S-POD data so far [12-19]. As experimentally demonstrated in Sec. IV, loworder instabilities that demand particular attention are mostly 1D; primary resonance bands accompanied by serious particle losses run horizontally and vertically on the tune diagram (cf. Fig. 10) unless non-negligible nonlinear fields are intentionally or accidentally introduced $[12,13]$.

In reality, artificial imperfection fields are unavoidable, which adds extra driving terms to $H_{0}$. The exact Hamitonian is

$$
H=H_{0}+\Delta U_{\text {ext }}(x, y ; s),
$$

where $\Delta U_{\text {ext }}(x, y ; s)$ is the external nonlinear potential due to mechanical errors and correction magnets. If the perturbation $\Delta U_{\text {ext }}$ is periodic with respect to $s$, it could substantially enhance nonlinear collective resonances. According to Ref. [25], the $m$ th-order collective mode in the horizontal or vertical direction can be driven by the $j$ thorder imperfection if $j \geq m$ and $j+m=$ even [35]. The condition of this external-field-driven resonance is given by

$$
\Omega_{m} \approx n
$$

that can be converted into the form

$$
m\left(\sigma_{0}-C_{m} \Delta \bar{\sigma}\right) \approx 2 n \pi .
$$

Equation (8) was first derived in the pioneering work by Sacherer who analytically solved the 1D Vlasov-Poisson equations under the smooth approximation $[22,26]$. The growth rate of the instability under the condition (8) depends on the strength of the error fields as well as on the beam intensity. This resonance is, therefore, of practical importance even at low intensity unlike the self-field-driven resonance under the condition (5) that disappears at the zero-intensity limit.

Except for the dipole mode $(m=1)$, the factor $C_{m}$ is in the range between 0.75 and $1.0[22,25]$. The magnitude of a coherent band shift is, therefore, not extremely sensitive to 
the resonance order $m$. Since $C_{m}<C_{m^{\prime}}$ for $m<m^{\prime}$, the band shift of a higher-order mode is somewhat greater while the band width narrower. Theoretically, many resonances that have the same $n / m$ ratio in Eqs. (5) and (8) more or less overlap. Needless to say, the external-fielddriven and self-field-driven resonances discussed here are collective phenomena, thus nothing to do with incoherent single-particle mechanism [36]. They can be treated simultaneously with the help of the Vlasov formalism. The Hamiltonian $H$ in Eq. (6) contains all physical information required for a general description of both types of collective resonances. If it is possible to solve Eqs. (2) and (3) mathematically with $H$ instead of $H_{0}$, the resultant universal solution will predict the horizontal or vertical 1D instability of the $m$ th-order mode under the condition just like Eq. (4). Then, for even $n$ that leads to the condition (7), the stop-band width and growth rate will be a function of not only the space-charge intensity but also imperfectionrelated parameters [25]. For odd $n$, the instability will be free from the latter parameters. We shall show later in an appendix that the coherent resonance condition (5) may naturally be extended to two-dimensional (2D) coupling resonances [12].

\section{B. Numerical simulations}

Before proceeding to experimental observations, let us take a look at some typical numerical results for later reference. We have done a number of particle-in-cell (PIC) simulations to support our interpretations of S-POD data [12-19]. The WARP code has been employed for this purpose [37]. Figure 2 shows WARP results obtained with the symmetric FODO waveform for $K_{\mathrm{QM}}(s)$. The longitudinal degree of freedom has been ignored to save computing time. The initial particle distribution is in the thermally equilibrated state well matched to the external focusing potential. The WARP code can carry out the sophisticated self-consistent matching to an AG lattice including the Debye screening effect [38]. What particle species we choose does not matter. The resonance condition is free from such information. The most essential information are the focusing strength and beam density, in other words, the bare phase advance $\sigma_{0}$ and tune depression $\eta$. The PIC simulations in Fig. 2 have assumed a proton beam traveling along the FODO channel at the kinetic energy of $1 \mathrm{MeV}$, but these parameters are physically of no importance. We obtain substantially the same emittance-growth curve as in Fig. 2 for any ion species at any beam energy (within a range of numerical error) as long as the initial tune depression is fixed. Since no error fields have been included here, the observed rms emittance growth comes purely from self-field-driven resonances; the results in Fig. 2 reflect what we expect in the ideal dynamical system govern by the Hamiltonian (1).

At zero intensity, no emittance growth occurs over the whole tune range. Switching the Coulomb potential on, we

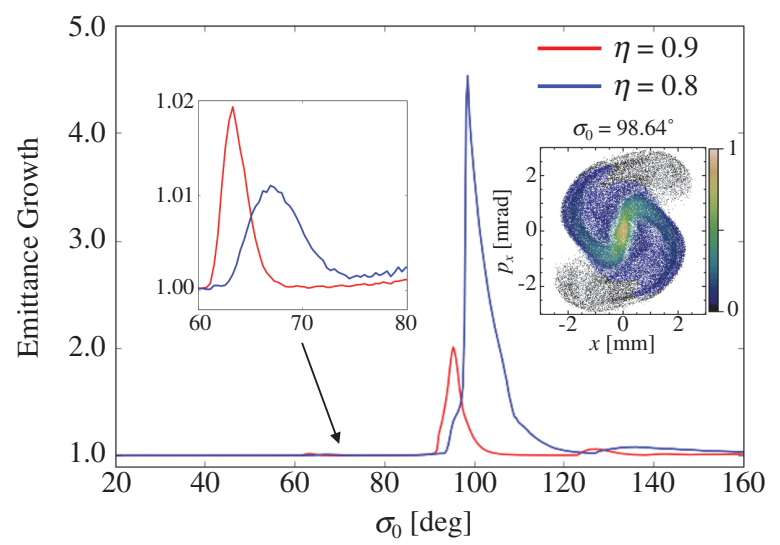

FIG. 2. WARP simulation results assuming the symmetric FODO lattice with $\xi=0.5$ and $\zeta=1$. The external focusing potential is completely linear (no nonlinear error fields). The growth rate of average transverse rms emittance after 100 FODO periods is plotted as a function of the bare betatron phase advance $\sigma_{0}$. We have initially taken a $1 \mathrm{MeV}$ proton beam in the thermal equilibrium state well matched to the FODO lattice. Two different initial tune depressions are considered here; namely, $\eta=0.8$ (blue) and 0.9 (red). The final phase-space profile at $\sigma_{0}=98.64^{\circ}$ in the case of $\eta=0.8$ is displayed which supports our expectation that the linear resonance $(m=2)$ is responsible for the large emittance growth. The color scale attached represents the normalized particle density. The $90 \%$ emittance assumed at the entrance of the transport channel is $0.98 \pi \mathrm{mm} \cdot \mathrm{mrad}$. A different choice of the input emittance only changes the scales of the $\left(x, p_{x}\right)$-axes, keeping the overall phase-space configuration. The growth rate is also unaffected as long as $\eta$ is fixed.

find a couple of instability regions above $\sigma_{0}=90^{\circ}$ and $120^{\circ}$. In addition to these two regions, there is another very weak instability growing near $\sigma_{0}=60^{\circ}$. All of them move rightward in Fig. 2 as the betatron motion is more depressed by the Coulomb potential. At high intensity, the instability near $\sigma_{0}=90^{\circ}$ becomes far more severe than the other two. The linear self-field-driven resonance $(m=2)$ is responsible for this major emittance growth as evidenced by the phase-space plot in Fig. 2. It corresponds to the well-known collective mechanism sometimes referred to as envelope instability $[4,24,28-32]$. While the condition (5) predicts the existence of other even-order $(m=4,6,8, \ldots)$ resonances overlapping, it is illogical to insist that any of these higher-order nonlinear resonances can dominate over the linear instability in the absence of external nonlinear driving forces. The Vlasov theory actually says that the growth rate of a lower-order resonance is higher without error fields [25]. The same argument applies to other instabilities identified near $\sigma_{0}=60^{\circ}$ and $120^{\circ}$; in practice, we should be more cautious about the third-order mode $(m=3)$ rather than the higher-orders' $(m=6,9, \ldots)$. The self-field-driven instabilities of highly nonlinear modes will be weakened or totally suppressed by the Landau-damping mechanism. Note that the coherent dipole $(m=1)$ resonance is located at the edge of the stability domain, i.e., at 
$\sigma_{0}=180^{\circ}$, and thus never excited under the present lattice condition. Detailed results of S-POD experiment on the dipole instability have been given in Ref. [19].

Coherent resonances overlapping at a certain operating point are not necessarily excited at the same timing. Our WARP simulations have revealed that the higher-order resonance tends to be activated earlier within an instability region where two resonances of relatively low orders overlap. This tendency has also been reported in another numerical work based on a different PIC code [39]. In the region just above $\sigma_{0}=90^{\circ}$, we often see the excitation of the weak octupole instability $(m=4)$ first followed by the quadrupole $(m=2)$. The latter instability is so strong that it quickly destroys the original matched beam core before the 100th cell even at the tune depression of 0.8 (cf. the phasespace plot in Fig. 2). Similarly, the weak sixth-order instability could be manifested prior to the third-order instability in the vicinity of $\sigma_{0}=60^{\circ}$. The very small emittance growth near $\sigma_{0}=60^{\circ}$ in Fig. 2 is probably due to the preceding sixth-order resonance because, unlike the quadrupole resonance at $\sigma_{0} \approx 90^{\circ}$, the transport over 100 FODO periods seems too short for the sextupole mode $(m=3)$ to grow sufficiently at the beam intensity considered here. Longer-term WARP simulations with the waterbag beam point out that the self-field-driven sextupole resonance needs hundreds of FODO periods to get excited when $\eta=0.8$. Figure 3(a) shows the phase-space evolution at $\sigma_{0}=69.12^{\circ}$. The three-arm structure peculiar to the third-order instability has been developed after the 400th cell. Before then, nothing significant happens at this operating point. The timing when the emittance starts to blow depends not only on the tune depression but also on the bare phase advance. At a slightly higher $\sigma_{0}$, we first observe a small emittance growth before 400th cell due to the sixth-order resonance that is eventually taken over by the sextupole instability. An example is displayed in Fig. 3(b) where the operating point has been shifted from $\sigma_{0}=69.12^{\circ}$ to $70.92^{\circ}$. The emittance evolution appears to have two qualitatively different stages. The core distribution in the early stage suggests the activation of weak sixthorder instability. The six-arm structure is smeared out before the second stage where the sextupole-looking instability becomes active causing a relatively rapid emittance growth. Such a transition from a weak high-order instability to a more dominant low-order instability is prone to occur on the high-tune side of a wide instability band. This is consistent with the coherent resonance condition in Eq. (5) or (8) predicting that the phase-shift factor $C_{m}$ is greater for a higher-order mode.

In S-POD, the radio-frequency (rf) potential for transverse plasma confinement inevitably includes weak nonlinearity originating from mechanical imperfections, e.g., slight electrode misalignments. Strictly speaking, the dynamical system emulated by S-POD obeys the Hamiltonian (6) rather than the Hamiltonian (1) (so do
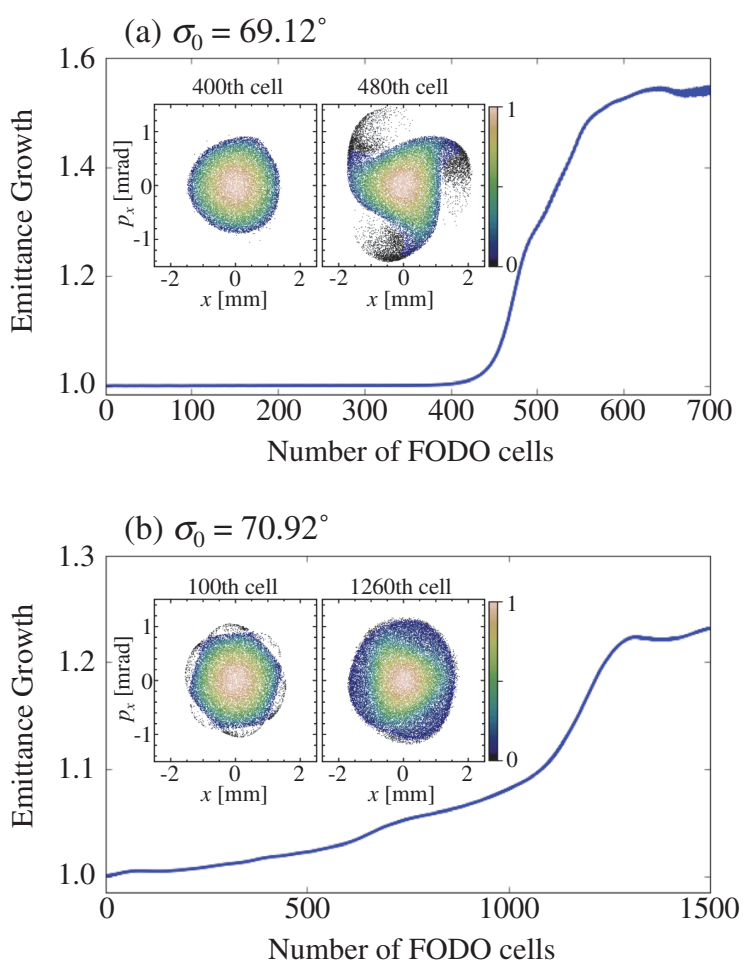

FIG. 3. Transverse rms emittance evolution of a waterbag beam propagating through a long FODO channel with $\xi=0.5$ and $\zeta=1$. The initial tune depression is set at $\eta=0.8$ in both examples. The bare phase advance in the upper picture (a) is $\sigma_{0}=69.12^{\circ}$ while in the lower picture (b), the operating point is slightly moved to $\sigma_{0}=70.92^{\circ}$ within the same local instability band. The external potential includes no nonlinearity. Two insets in each picture exhibit the horizontal phase-space profiles after the waterbag beam has passed through certain numbers of FODO cells; namely, the profiles at the 400th cell and at the 480th cell in the upper picture, and the profiles at the 100th cell and at the 1260th cell in the lower. Following the PIC simulations in Fig. 2, we have assumed a $1 \mathrm{MeV}$ proton beam with the initial emittance $(90 \%)$ of $0.88 \pi \mathrm{mm} \cdot \mathrm{mrad}$.

real beam transport channels). The external nonlinear potential $\Delta U_{\text {ext }}$ is generally much weaker than the quadrupole focusing potential but for sure nonzero depending on how precisely the LPT was fabricated [17,40]. In a regular LPT, the error fields are turned on simultaneously with the linear focusing field, so $\Delta U_{\text {ext }}$ has the same periodicity as $K_{\mathrm{QM}}(s)$ and may expedite the nonlinear resonance process. Such a driving-force condition peculiar to the LPT has been taken into account in the numerical results of Fig. 4. We have incorporated periodic imperfection fields in these WARP simulations, assuming possible misalignments of the four quadrupole rods. The strengths of error-induced nonlinear multipole components are less than $1 \%$ of the quadrupole strength [40]. The sextupole amplitude, which is the largest among all nonlinear components, is roughly $0.36 \%$ for the rms electrode misalignments of $0.05 \mathrm{~mm}$. The strengths of all multipole components are approximately doubled with the average misalignments of $0.1 \mathrm{~mm}$. 
(a) $\eta=0.9$

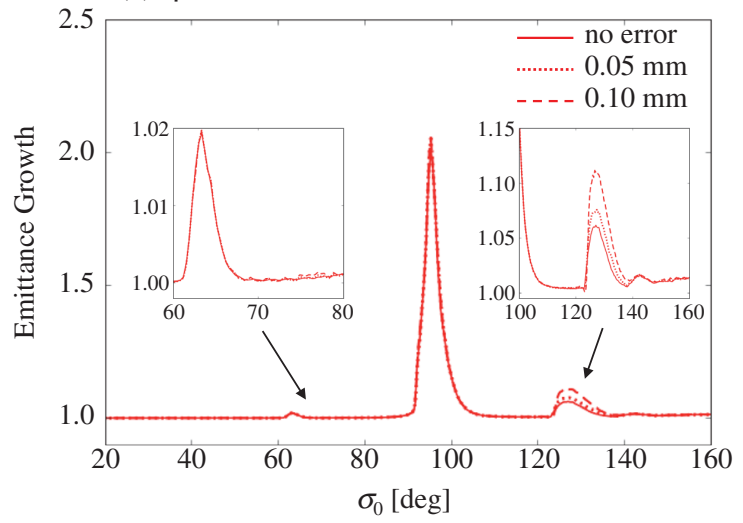

(b) $\eta=0.8$

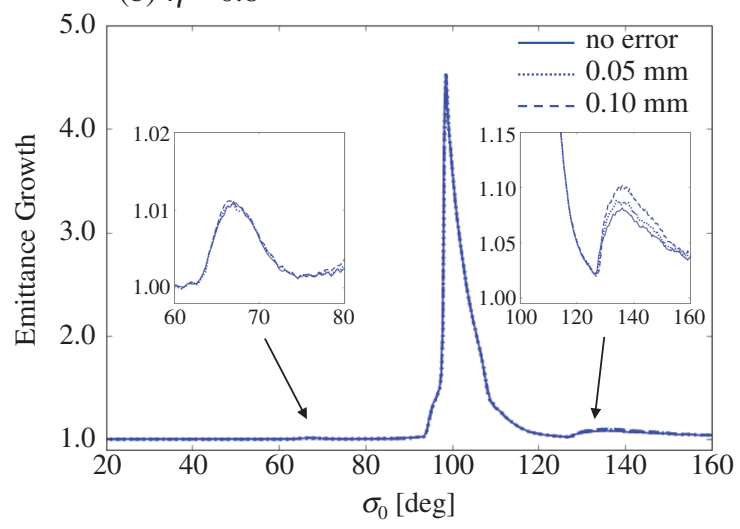

FIG. 4. WARP simulation results in consideration of the rf-field property of the LPT. Nonlinear imperfection fields up to the sixth order have been introduced in these PIC simulations. The strengths of the nonlinear fields are determined by analyzing the weak rf-potential distortion caused by misalignments of the four quadrupole rods. The upper picture corresponds to the case where $\eta=0.9$ while the lower to the case where $\eta=0.8$. In each picture, we have considered two different sizes of rms misalignments; namely, $0.05 \mathrm{~mm}$ (dotted line) and $0.1 \mathrm{~mm}$ (broken line). The result in Fig. 2 under the ideal linear focusing condition is replotted with solid line for reference. The average nonlinear multipole strengths relative to the quadrupole's are listed in Table 1 of Ref. [40] and assumed in these simulations. The rf focusing waveform used for the transverse confinement of ${ }^{40} \mathrm{Ar}^{+}$ ions is the symmetric FODO with $\xi=0.5$ and $\zeta=1$. The initial distribution is in the thermal equilibrium and well matched to the AG potential. The ordinate represents the growth rate of average transverse rms emittance after $0.1 \mathrm{~ms}$ corresponding to 100 FODO periods. The effect of image charges induced on the electrode surfaces has been incorporated for completeness but it turns out to be negligible according to separate test simulations without the image effect.

We recognize that the plasma instability above $\sigma_{0}=120^{\circ}$ is strengthened by the mechanical error in both cases (a) and (b) where $\eta=0.9$ and 0.8 respectively. The effect of the error fields on other two stop bands turns out to be very weak at least within 100 FODO periods. There is no clear signature of additional resonances, despite that the external

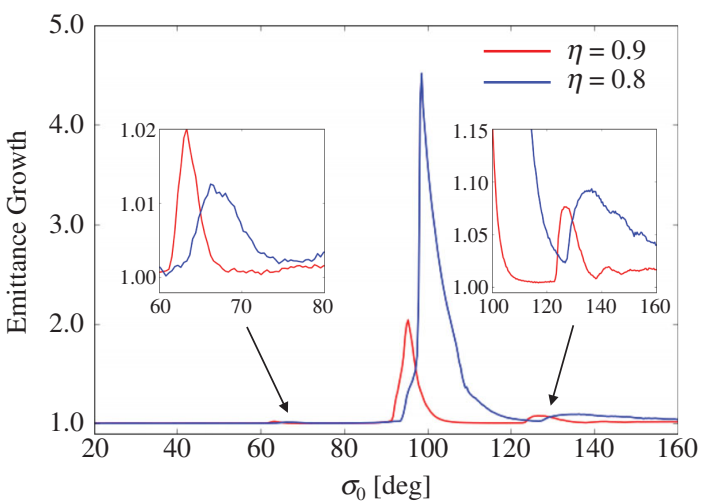

FIG. 5. WARP simulation results in consideration of random nonlinear error fields. The initial conditions are equivalent to those in Fig. 2, except for the inclusion of random nonlinearities in quadrupole magnets to emulate the ordinary linear beam transport situation. The absolute strengths of high-order multipole components in each focusing element are defined by using uniform random numbers within the range from 0 to 2 , such that their average values over 100 FODO cells (200 Q-magnets) agree with the strengths of the periodic nonlinearities assumed in Fig. 4 for the case of the rms misalignment of $0.05 \mathrm{~mm}$.

driving potential has included finite nonlinearities up to the sixth order.

A question now is whether external error fields exert any influence on nonlinear resonance bands even if they are not exactly periodic. This is the case in actual linear transport channels where the beam orbit is not closed. The question is answered in Fig. 5 where we have assumed random nonlinear imperfections along the channel. The absolute strengths of the imperfection fields relative to the linear focusing field are set equivalent on average to the case of 0.05 -mm rms misalignment in Fig. 4. No essential change can be seen depending on whether the error fields hold perfect periodicity (Fig. 4) or not (Fig. 5).

\section{BEAM-DYNAMICS MODELING WITH S-POD}

Since the information of S-POD has been given in many previous publications [14-20,41,42], we only briefly outline how it works. As already mentioned above, S-POD provides a compact many-body system that approximately obeys the Hamiltonian

$$
\begin{aligned}
H_{\mathrm{S}-\mathrm{POD}}= & \frac{p_{x}^{2}+p_{y}^{2}}{2}+\frac{1}{2} K_{\mathrm{rf}}(\tau)\left(x^{2}-y^{2}\right) \\
& +I_{\mathrm{p}} \phi+\Delta U_{\mathrm{rf}}(x, y ; \tau),
\end{aligned}
$$

where the independent variable is now time $\tau=c t$ with $c$ being the speed of light, $I_{\mathrm{p}}$ is a constant associated with the species of confined particles, $K_{\mathrm{rf}}(\tau)$ is a periodic function proportional to the $\mathrm{rf}$ focusing voltages $V_{\mathrm{rf}}(\tau)$ on the quadrupole rods, and $\Delta U_{\mathrm{rf}}(x, y ; \tau)$ represents the nonlinear potential that comes from mechanical imperfections and 
has the same periodicity as $K_{\mathrm{rf}}(\tau)$ [43]. Similarly to the beam transport system, the Coulomb potential $\phi$ in Eq. (9) is determined by solving the Poisson and Vlasov equations in the self-consistent manner. Since $\Delta U_{\mathrm{rf}}$ is weak in a regular LPT, the Hamiltonian (9) is very close to $H_{0}$ in Eq. (1). Our experimental model is physically equivalent to the theoretical basis frequently adopted for diverse spacecharge studies in the accelerator community.

There are many practical advantages in S-POD experiment, compared with conventional approaches to intense beam issues. First of all, S-POD is far more compact and incomparably cheaper than any accelerators. Thanks to the compactness and simple nature of the system, we have only little noise sources that complicate output signals. It is thus possible to explore collective effects expected in the very basic model of Eq. (9). Second, S-POD is extremely flexible in controlling fundamental beam-dynamics parameters. For instance, the waveform of the AG focusing function $K_{\mathrm{rf}}(\tau)$ can be changed over a very wide range without any mechanical modifications to the LPT structure. Since $K_{\mathrm{rf}}(\tau)$ is proportional to the rf voltages applied to the quadrupole rods, all we need for the study of latticedependent effects is simply to modify the rf waveform from the power supply. In principle, we can emulate any AG focusing function [44]. Third, S-POD is not a numerical simulation tool but an experimental apparatus. Our approach is free from drastic approximations and assumptions often made in computer codes to reduce CPU time. Each experimental simulation run with S-POD at a particular operating tune is completed almost instantly no matter whether the beam intensity is high or low. In addition, we do not have to worry about radio-activation due to the instant loss of a whole intense bunch because the energy of stored particles is very low in the laboratory frame. Taking these facts seriously, the Intense Beams Group of Accelerator Science and Technology Centre in England recently decided to construct a LPT system closely following the S-POD design [45]. The English ion-trap facility, named "IBEX" (Intense Beam Experiment), is now in the middle of commissioning. There is another dedicated ion trap at Princeton Plasma Physics Laboratory in the US. Gilson and his co-workers have done interesting beamphysics experiments, using a different type of LPT [46-48]. Their work includes a detailed study of beam-quality degradation induced by white and colored noises on the linear focusing function $K_{\mathrm{rf}}(\tau)$ [49,50].

Our LPT is roughly $200 \mathrm{~mm}$ long and divided into five independent sections so that two axial potential wells can be formed if necessary by giving different DC bias potentials to those sections [41]. Since the plasma confinement is static in the longitudinal direction, we are not bothered by synchrotron resonance under the current S-POD setup (while it is possible to excite such resonance by adding a periodic modulation in the axial confinement force [51]). A microchannel plate (MCP) with a phosphor screen is placed about $20 \mathrm{~mm}$ away from one end of the LPT, and on the other side, we have a Faraday cup (FC) detector. ${ }^{40} \mathrm{Ar}^{+}$ions produced through the electron bombardment process are used for the experiment. The repetition frequency of the doublet waveform in Fig. 1 is set at $1 \mathrm{MHz}$. Then, the maximum step height required for the full tune-space survey is only $101.9 \mathrm{~V}$ for the symmetric FODO lattice [42]. We introduce neutral Argon gas atoms into the chamber and ionize them with a low-energy coasting electron beam from an e-gun. Generated ions are accumulated within a circular boundary of $5 \mathrm{~mm}$ in radius surrounded by the four quadrupole rods. The transverse extent of the non-neutral plasma is a few $\mathrm{mm}$ [19] while the axial extent is much larger, i.e., roughly $50 \mathrm{~mm}$, to emulate a long bunch. As a future subject, we are considering the use of a short bunch (with or without the driven axial resonance) to investigate the effect of synchrobetatron coupling [52].

It takes about a second to accumulate a sufficient number of ${ }^{40} \mathrm{Ar}^{+}$ions. The initial plasma density can be controlled by changing the Argon-gas pressure and/or the electronbeam current. The base vacuum pressure is $1 \times 10^{-8} \mathrm{~Pa}$, which is worsened to the order of $10^{-5} \mathrm{~Pa}$ after the introduction of the neutral gas when we need a highdensity plasma (cf. Ref. [21]). The tune depression reached with $10^{7}$ stored ions in the LPT is estimated to be about $0.85-0.9$ [16]. After an ion bunch is formed, we shut down the electron beam, wait typically for $50 \mathrm{~ms}$ until the bunch settles into a sort of stationary state, and then adjust the LPT operating point to a proper intended position at which the bunch is maintained for a certain period to test its stability. The operating betatron tunes are evaluated at high accuracy (within an error of $\pm 0.1 \%$ ) through direct measurement of the rf voltages on the electrodes. Finally, the axial potential barrier on either the MCP or FC side is dropped to extract the ions. If the bunch is unstable at the chosen operating point due to the transverse coherent resonance, we will detect a noticeable reduction of the output ion signal. The single measurement cycle at a particular operating point is finished within $10 \mathrm{~s}$ including the whole data transfer and saving on a personal computer. The reproducibility of S-POD data is very good. Since the experimental procedure is automated, we do not have to stay beside S-POD to retune fundamental parameters. Only less than one hour is enough for several hundred measurements executed at several hundred different operating points at any density.

At present, there are three independent LPT-based S-POD systems (S-POD I, II, and III) operating side by side at the Beam Physics Laboratory in Hiroshima. We can double-check experimental data whenever necessary. The mechanical designs of the three LPTs are similar, but of course, they have different nonlinear characters due to the random nature of errors in electrode machining and assembling. We expect the rms average of electrode misalignments to be around $50 \mu \mathrm{m}$ to $100 \mu \mathrm{m}$ at most. 
Previous experimental observations indicate that the mechanical imperfection is the largest in the LPT currently used for S-POD III [53]. In fact, it is always easier for us to observe nonlinear effects in S-POD III, compared with the other two systems $[12,14]$. This study was done with S-POD II where the error-induced nonlinearities are relatively weak. Throughout the paper (except for Appendix C), the plasma storage period at each operating point is fixed at $10 \mathrm{~ms}$ corresponding to 10,000 FODO cells. It should be long enough to find out practically important instabilities that might cause non-negligible emittance growth in real linear transport channels.

\section{RESULT}

\section{A. FODO and sinusoidal focusing}

In previous S-POD experiments as well as WARP simulations [14-19], we have often assumed the sinusoidal focusing potential commonly used for regular LPTs while the FODO waveform was employed in some earlier iontrap experiments [47,54]. Theoretically, the sinusoidal focusing channel has a resonance feature very similar to that of the FODO channel. Figure 6 shows the ion-loss distributions under the sinusoidal focusing potential. Three clear instability regions repeatedly observed in past S-POD experiments have appeared here again. The instabilities are located near $\sigma_{0}=60^{\circ}, 90^{\circ}$, and $120^{\circ}$, all of which shift to the higher-tune side as the initial number of stored ions is increased. This observation qualitatively agrees with the PIC simulation results in Sec. II. Particular attention should be paid to the following facts. First, ion losses at $\sigma_{0} \approx 60^{\circ}$ and $90^{\circ}$ are very weakened in the low-intensity regime, which suggests that the primary source of the instability is

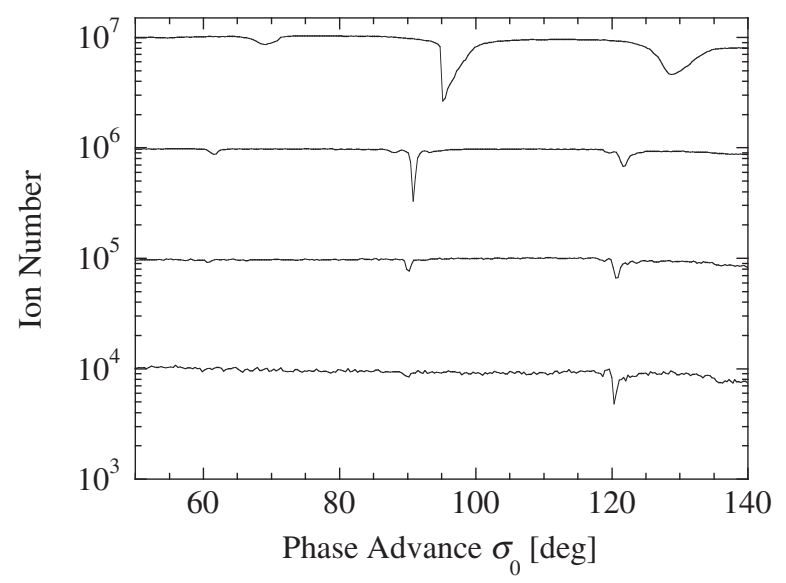

FIG. 6. Ion-loss distributions measured in S-POD with the sinusoidal focusing potential. Each of the four curves consists of 237 data points obtained from 237 independent measurements over the range $50^{\circ} \leq \sigma_{0} \leq 140^{\circ}$ at the same initial ion number $\left(N_{\text {ion }} \approx 10^{4}, 10^{5}, 10^{6}\right.$, or $\left.10^{7}\right)$. The storage period of ions in the LPT is fixed at $10 \mathrm{~ms}$ in all four cases. It takes us only less than 40 minutes to complete 237 measurements. the Coulomb self-field potential. Second, unlike these two instability bands, ion losses near $\sigma_{0}=120^{\circ}$ remain observable even at very low density. The lowest-order instability expected from the condition (8) in the range $\sigma_{0}<180^{\circ}$ is the sextupole $(m=3)$ resonance that occurs at $\sigma_{0} \approx 120^{\circ}$. The third-order error field must, therefore, be playing a central role around this phase advance. Third, the instability at $\sigma_{0} \approx 90^{\circ}$ rapidly grows with increasing number of initial ions and becomes most severe in the high-intensity regime. The lowest-order mode that can be unstable near $\sigma_{0}=90^{\circ}$ is the quadrupole $(m=2)$ and the second lowest is the octupole $(m=4)$. The former is driven by the Coulomb potential and the latter not only by the Coulomb potential but also by the fourth-order external field depending on the degree of mechanical imperfections. Recalling the PIC simulation results in Sec. II B and observed instability suppression in the low-intensity regime, we come to a conclusion that serious ion losses near $\sigma_{0}=90^{\circ}$ at high density should be due mainly to the self-field-driven linear resonance. A similar argument applies to the origin of the small dip at $\sigma_{0} \approx 60^{\circ}$; the most straightforward interpretation is that ion losses there are caused largely by the selffield-driven sextupole $(m=3)$ resonance rather than the much higher-order instability of the $m=6$ mode.

The stop-band behavior almost identical to what we found in Fig. 6 has been confirmed experimentally with the doublet lattice $[12,13]$. The result in Fig. 7 verifies that the basic feature of the ion-loss distribution does not change even if the rf waveform for $K_{\mathrm{rf}}$ is switched from the sine curve to the symmetric FODO configuration with $\xi=0.5$ and $\zeta=1$. We encounter the three instability bands that have the same characteristics as those discovered in Fig. 6.

It is well known from rms envelope theories that the instability near $\sigma_{0}=90^{\circ}$ may involve the resonances of two distinct modes referred to as "in-phase (breathing)" and "out-of-phase (quadrupole)" modes [4]. In S-POD

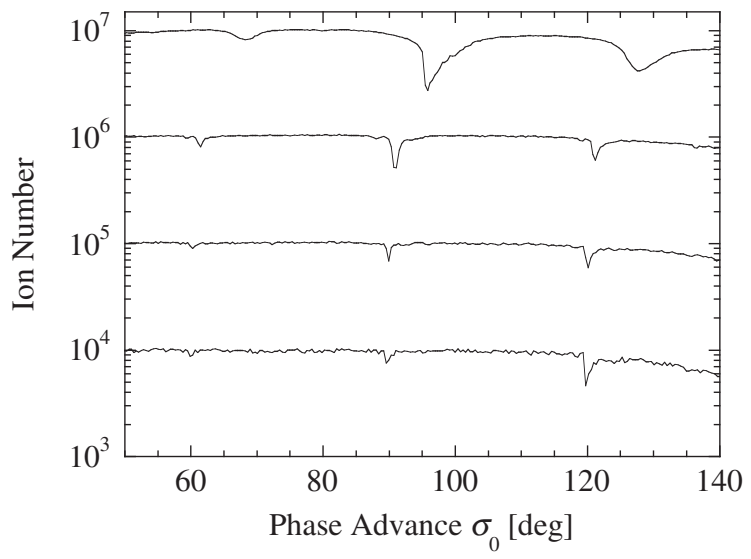

FIG. 7. Ion-loss distributions measured in S-POD with the symmetric FODO potential where $\xi=0.5$ and $\zeta=1$. The experimental condition is the same as adopted in Fig. 6 except for the rf focusing waveform of $K_{\mathrm{rf}}(\tau)$. 
experiment, however, no clear evidence that proves this theoretical prediction has been obtained so far. Ion losses above $\sigma_{0}=90^{\circ}$ always form a single pronounced dip at high density as displayed in Figs. 6 and 7. This seems reasonable because the external driving field in the LPT is free from the axisymmetric component that can directly drive the breathing oscillation. A careful analysis of the envelope equations by Lund and Bukh has revealed that in quadrupole doublet channels, the two linear eigenmodes lose stability within almost the same ranges of parameters [30]. It is, therefore, practically impossible to separate these envelope instabilities in S-POD.

\section{B. Dependence on the geometric factors}

It is not surprising that the stop-band distributions of the FODO and sinusoidal focusing channels have no striking difference [15]. In fact, the matched envelope modulations along these two AG channels are nearly identical. The result in the last subsection makes us anticipate that analogous stop-band behavior must be observed in a Focus-Defocus-Defocus-Focus (FDDF) transport channel as well. The FDDF lattice has been adopted in some major machines such as the Proton Synchrotron (PS) at CERN [9] and the UNILAC at GSI [7]. An example of S-POD data verifying this natural expectation is given in Appendix A.

The drift-space ratio $\zeta$ is not necessarily equal to 1.0 as assumed in Fig. 7. Moreover, the quadrupole filling factor $\xi$ is different from 0.5 in most of existing beam transport lines. It is thus valuable to examine whether the choice of the geometric factors has any influence on the "three-band structure" in Fig. 7. Such an experimental study, generally impossible in a large-scale machine that has a unique uncontrollable lattice configuration, can be conducted very easily in S-POD. The right panels in Figs. 8 and 9 show the ion-loss distributions when the rf waveforms as in the left panels are employed for $K_{\mathrm{rf}}(\tau)$. The quadrupole filling factor $\xi$ is changed in Fig. 8 with the drift-space ratio $\zeta$ fixed at 1.0. The familiar stop bands have reappeared at $\sigma_{0} \approx 60^{\circ}, 90^{\circ}$, and $120^{\circ}$ regardless of the change in $\xi$. Essentially the same distribution is found in Fig. 9 where we have varied $\zeta$ instead of $\xi$. Previous WARP simulations have pointed out a possible correlation between the $\zeta$-parameter and instability growth rate [15], but the effect looks too weak to be identified in Fig. 9. These observations allow us to conclude that the global resonance picture is unaffected by variation in the geometry factors.

The envelope theory in Ref. [30] disclosed a thin stop band overlooked in earlier work on the linear collective modes. The stop band, named "lattice resonance band" in Ref. [30], is manifested when $\zeta \neq 1$. It is, however, very narrow and located very close to the major instability region at $\sigma_{0} \approx 90^{\circ}$, which makes it difficult for us to confirm its existence under the current operating condition of S-POD. For experimental verification of the lattice
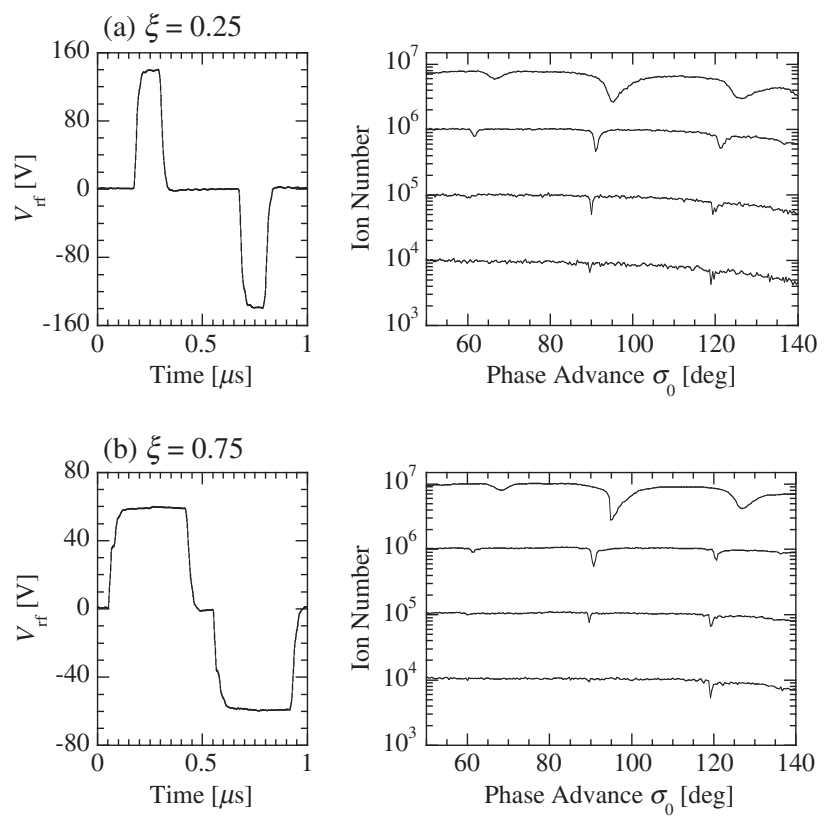

FIG. 8. Ion-loss distributions measured in S-POD with doublet focusing waveforms of different quadrupole filling factors. The drift-space ratio is fixed at $\zeta=1$ while we consider two quadrupole filling factors: (a) $\xi=0.25$ and (b) $\xi=0.75$. The left panels show the rf waveforms generated by the S-POD power supply when $\sigma_{0}$ is adjusted to $90^{\circ}$. The storage period of ions in the LPT is fixed at $10 \mathrm{~ms}$.
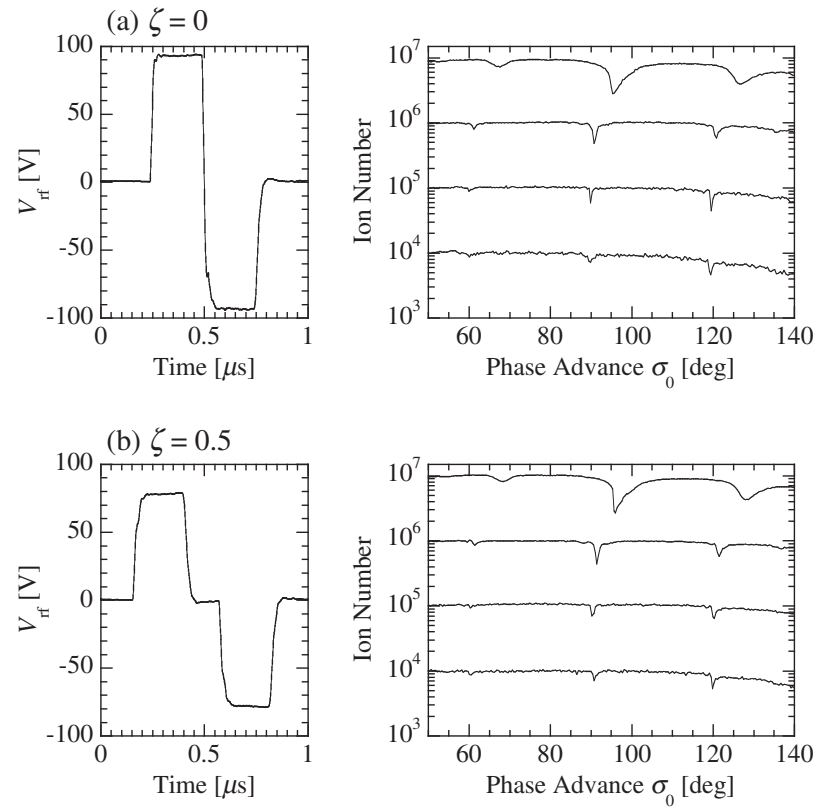

FIG. 9. Ion-loss distributions measured in S-POD with doublet focusing waveforms of different drift-space ratios. The quadrupole filling factor is fixed at $\xi=0.5$ while we consider two driftspace ratios: (a) $\zeta=0$ and (b) $\zeta=0.5$. The left panels show the rf waveforms generated by the S-POD power supply when $\sigma_{0}$ is adjusted to $90^{\circ}$. The storage period of ions in the LPT is fixed at $10 \mathrm{~ms}$. 
resonance band, we may need to try further increasing the initial plasma density, so that the thin stop band is sufficiently distanced from the dominant $90^{\circ}$ band.

\section{Asymmetric focusing}

The experiments and numerical simulations in the previous sections have assumed the symmetric focusing condition $\sigma_{0 x}=\sigma_{0 y}\left(\equiv \sigma_{0}\right)$ where $\left(\sigma_{0 x}, \sigma_{0 y}\right)$ are the horizontal and vertical bare betatron phase advances per unit cell. The two transverse phase advances are often set equal or close to each other in drift tube linacs (DTLs) [55,56]. For instance, the DTLs at some major Japanese institutes including J-PARC, RIKEN, and NIRS have actually used the FODO lattice under the condition $\sigma_{0 x}=\sigma_{0 y}$ [57]. Most of past theoretical studies of intense beam transport have also assumed symmetric transverse focusing [4,24,28-32]. As pointed out later in this section, the coupling resonance along the line $\sigma_{0 x}-\sigma_{0 y}=0$ on the tune diagram is practically harmless to an equilibrium beam that has the same emittances in both directions. Choosing the operating point $\sigma_{0 x}=\sigma_{0 y}$, therefore, seems quite natural because a round beam is certainly preferred for typical drift tubes with a small circular aperture.

In this subsection, we take a step forward to explore the general case where $\sigma_{0 x} \neq \sigma_{0 y}[12,13,15]$. The transverse net focusing forces in the LPT can readily be made asymmetric by controlling the rf waveform for $K_{\mathrm{rf}}(\tau)$. Specifically, we alter the widths of the focusing and defocusing pulses in Fig. 1 separately to differentiate $\sigma_{0 x}$ and $\sigma_{0 y}$. Over four thousand measurements with different combinations of $\sigma_{0 x}$ and $\sigma_{0 y}$ were carried out and summarized in Fig. 10. The number of ions surviving after $10 \mathrm{~ms}$ at a specific operating point has been represented by the shading of gray scale on the tune diagram. The ion survival rate is lower in darker operating regions. We immediately recognize three horizontal and three vertical lines along which considerable reduction of the survival rate has occurred. All these instability lines are more widened and move to the higher-tune side with increasing number $N_{\text {ion }}$ of initial ions. This observation is consistent with the stop-band behavior shown in Figs. 6-9 where $\sigma_{0 x}=\sigma_{0 y}$. In the case of symmetric focusing, the LPT operating point is somewhere on the straight line inclined at an angle of $45^{\circ}$ on the stability map in Fig. 10. The horizontal and vertical resonance bands intersect on the line, which means that the ion losses in Figs. 6-9 contain contributions from at least two instabilities induced independently in the different degrees of freedom.

As discussed in the subsections II B and IVA, the most conspicuous lines slightly above $\sigma_{0 x(0 y)}=90^{\circ}$ are attributed largely to the self-field-driven linear resonances excited in the two transverse directions and partly to the weak octupole resonances. Other four lines near $\sigma_{0 x(0 y)}=60^{\circ}$ and $120^{\circ}$ should be due mostly to the horizontal and vertical sextupole resonances. Since external nonlinear fields strengthen the sextupole instability along $\sigma_{0 x} \approx 120^{\circ}$ and $\sigma_{0 y} \approx 120^{\circ}$, these two lines could be most troublesome at low beam intensity if the lattice includes large mechanical errors. At high beam intensity, the instabilities along $\sigma_{0 x(0 y)} \approx 60^{\circ}$ and $\sigma_{0 x(0 y)} \approx$ $90^{\circ}$ also become unavoidable even in a perfectly constructed machine because the natural Coulomb potential can be the source of them. The $90^{\circ}$ resonance lines are particularly dangerous as is evident from Fig. 10(c).

In addition to the six 1D stop bands running horizontally and vertically in Fig. 10, we notice two faint coupling resonance lines when the initial plasma density is high $\left(N_{\text {ion }} \approx 10^{7}\right)$. These weak instability bands originate most likely from the third-order coupling terms $x^{2} y$ and $x y^{2}$ as they can be fitted by $2 \sigma_{0 x}-\sigma_{0 y} \approx 0$ and $\sigma_{0 x}-2 \sigma_{0 y} \approx 0$. Both lines are observed even in PIC simulations without external nonlinearities (see Appendix B). Other coupling resonance lines are invisible or unclear in Fig. 10, which
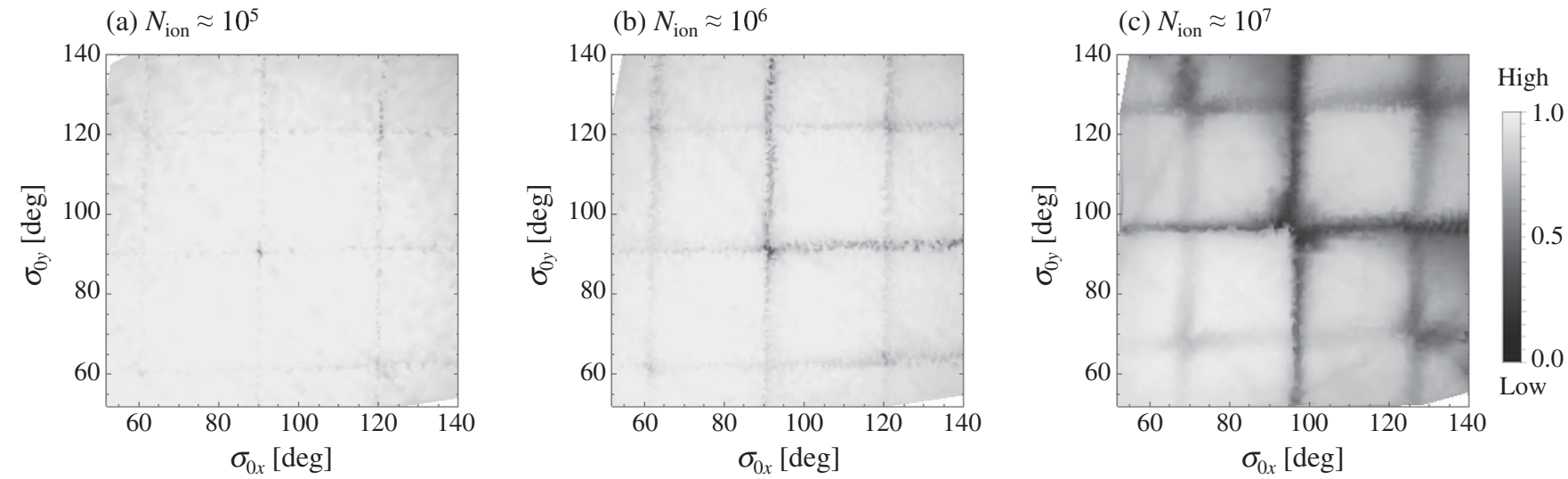

FIG. 10. Tune diagrams of the quadrupole doublet beam transport. The number of ions $\left(N_{\text {ion }}\right)$ initially stored in the LPT is about $10^{5}$ in the left picture (a), $10^{6}$ in the middle picture (b), and $10^{7}$ in the right picture (c). The storage period of ions in the LPT is fixed at $10 \mathrm{~ms}$ in all three cases. The rate of surviving ions is lower in the area of a darker shade. Each stability map is made from results of 4450 independent measurements performed with 4450 different combinations of $\sigma_{0 x}$ and $\sigma_{0 y}$. 
implies that mechanical imperfections are rather small in the LPT employed here for S-POD II. In another LPT for S-POD III, we can detect third-order, probably errorinduced coupling resonance lines more easily and clearly [12]. It was even possible to identify the fourth-order difference resonance line $2 \sigma_{0 x}-2 \sigma_{0 y}=0$. This type of difference resonance is usually undetectable not only due to the weakness of the fourth-order error field but also because the horizontal and vertical emittances are initially equal in a regular LPT when $\sigma_{0 x}=\sigma_{0 y}$. Since the sum of the two emittances is conserved under the resonance $2 \sigma_{0 x}-2 \sigma_{0 y}=0$, nothing remarkable happens if the plasma is isotropic in the transverse dimensions. We, therefore, used an elaborate plasma shaping technique to make the initial ion distribution anisotropic in the two transverse

(a) $N_{\text {ion }} \approx 10^{6}$

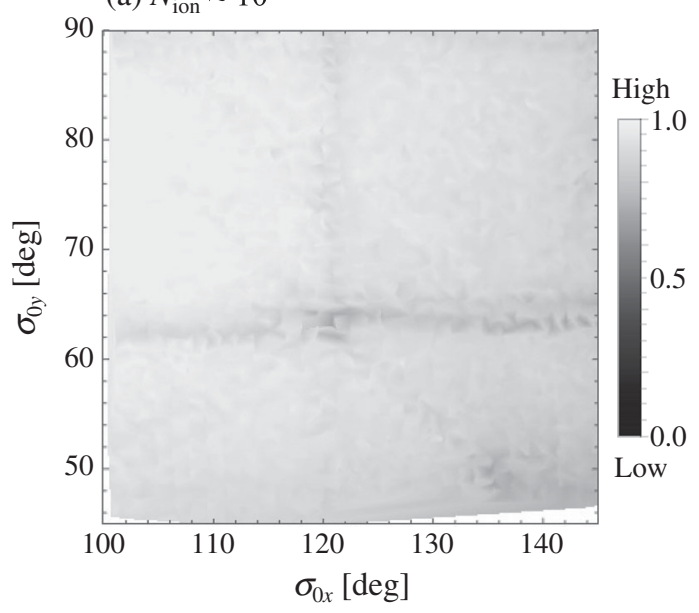

(b) $N_{\text {ion }} \approx 10^{7}$

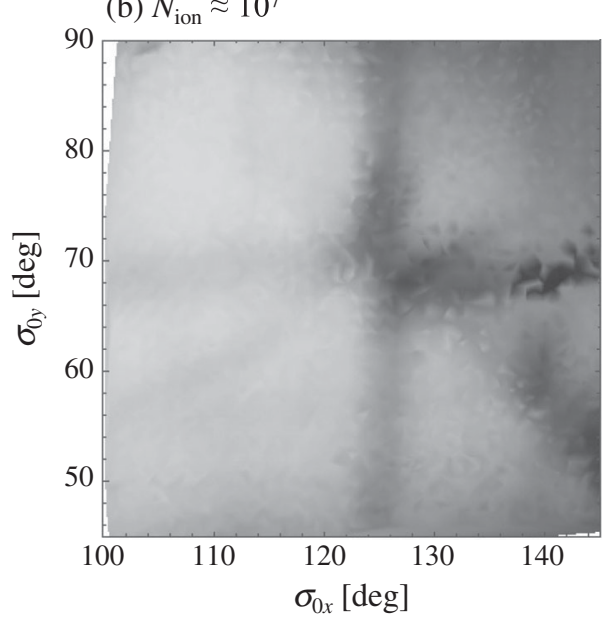

FIG. 11. Tune diagrams of the quadrupole doublet beam transport. The transverse tune space above $\sigma_{0 x}=100^{\circ}$ and below $\sigma_{0 y}=90^{\circ}$ in Fig. 10 is magnified to search for a weak coupling resonance line. The number of ions $\left(N_{\text {ion }}\right)$ initially stored in the LPT is about $10^{6}$ in the upper picture (a), and $10^{7}$ in the lower picture (b). The storage period of ions in the LPT is fixed at $10 \mathrm{~ms}$ in both cases. Each picture consists of 3969 data points. directions [58]. More nonlinear resonance lines have been successfully identified in S-POD III, but coupling instabilities of the fourth or higher orders are generally very weak and thus difficult to locate unless we considerably extend the period of plasma storage to enhance ion losses.

Boine-Frankenheim et al. recently pointed out the possibility of parametric sum resonance induced by the space-charge potential of second order [59]. Such a resonance line was not very clear in the original S-POD data of Fig. 10, so we focused our attention on a narrower area around $\left(\sigma_{0 x}, \sigma_{0 y}\right)=\left(120^{\circ}, 70^{\circ}\right)$ increasing the density of data points. Theoretically, the sum-resonance band is somewhat widened around this region, which gives us a better chance to find it out (cf. Appendix B). Figure 11 is an enlarged local tune map containing about 4000 data points. We now see a sum resonance band thicker than the thirdorder coupling resonance line. Although a further study is necessary to make a definitive conclusion, we presently suspect that the observed line is due to the skew-mode resonance discussed in Ref. [59]. It is possible to show that the Coulomb self-field potential can be a source of not only the linear sum resonance but also a variety of parametric coupling instabilities [60]. A plausible hypothesis is formulated in Appendix B regarding this issue.

\section{CONCLUDING REMARKS}

Systematic experiments have been conducted to investigate the stability of an intense hadron beam traveling in a periodic AG focusing channel. The Simulator of Particle Orbit Dynamics, the compact non-neutral plasma trap facility designed solely for diverse beam-physics applications, has been employed to clarify how the condition of coherent betatron resonance depends on fundamental parameters. For the last more than a decade, we have devoted a great deal of experimental and theoretical effort to this important subject, establishing the new tabletop research technique [12-20,25,40-42,51,60]. The high parameter controllability and flexibility available in S-POD experiments enable us to provide a definitive answer to the question of what operating condition should be avoided to minimize possible beam losses in a long AG transport line. The present study focuses on the FODO lattice (quadrupole doublet) that has been widely used as the most standard AG configuration for various machines. A key to the comprehensive understanding of our experimental results is the parametric resonance condition (5) first formulated in Ref. [25]. Only little attention has been paid to this instability criterion so far in spite of its practical importance at high beam density. It looks very similar to the Sacherer's famous coherent resonance condition (8) $[22,26]$, but the factor $1 / 2$ on the right-hand side of Eq. (4) yields essential difference in the interpretation of S-POD data [12-19]. The mathematical formulas given in Ref. [25] are not limited to the simple doublet focusing but applicable to arbitrary lattice configurations. The present 
discussion can readily be extended to the situation of highintensity storage rings $[12,14,16,19]$ (see Appendix C).

In the ordinary case where $\sigma_{0 x}=\sigma_{0 y}\left(\equiv \sigma_{0}\right)$, we have observed three instability bands near $\sigma_{0}=60^{\circ}, 90^{\circ}$, and $120^{\circ}$ not only in S-POD experiments but also in PIC simulations no matter whether weak nonlinear imperfection fields are present. As the initial beam density becomes greater, each stop band is broadened while moving its central position to the higher-tune side [61]. The global picture of ion losses is unchanged even in S-POD III that has relatively strong nonlinearities, compared with S-POD II used here $[14,16,18,19]$. In the high-intensity regime, the instability near $\sigma_{0}=90^{\circ}$ is especially serious producing much more ion losses than the other two. We have judged that the main cause of this severe instability is the linear $(m=2)$ self-field-driven resonance predicted by Eq. (5) [25] and earlier theoretical studies of collective oscillation modes $[4,24,28]$. The large stop band shrinks as the plasma density is lowered (see, e.g. Figs. 6 and 7). It almost disappears at very low density, which is in striking contrast with the instability near $\sigma_{0}=120^{\circ}$ driven primarily by the third-order $(m=3)$ imperfection field. If the fourth-order error in the LPT happened to be very enhanced somehow and thus created the most prominent stop band at $\sigma_{0} \approx 90^{\circ}$, the resultant ion losses would never vanish even in the lowintensity regime just like the $120^{\circ}$ stop band.

Similarly, we accept the most straightforward interpretation concerning the ion losses near $\sigma_{0}=60^{\circ}$; at high density, the self-field-driven resonance of the third-order mode rather than the sixth-order resonance plays a dominant role there in a long transport channel [12-17]. If we assume the ion losses in the vicinity of $60^{\circ}$ to be caused by the sixth-order instability, it seems difficult to explain the complete absence of the fifth-order resonance lines. Statistically speaking, a lower-order multipole field is stronger within the LPT aperture as long as electrode misalignments are completely random [40]. Besides, the $m$ th-order multipole potential is proportional to $(r / R)^{m}$ with $R$ being the aperture radius and $r$ being the distance from the LPT axis. Since the ratio $r / R$ is well below unity inside the beam core, the sixth-order effect should be much weaker than the fifth and lower orders'. As supported by PIC simulations in Sec. II, the sextupole instability does exist near the phase advance of $60^{\circ}$ and eventually gives rise to rms emittance growth more than that of the sixthorder-mode origin. Even though the emittance behavior is sensitive to several parameters such as the operating tune, beam density, and transport distance, it is misleading to claim that the sixth-order resonance can dominate over the self-field-driven third-order resonance when the beam is intense. According to our interpretation based on the criterion in Eq. (5), the three conspicuous instabilities experimentally observed at high density can be understood as the coherent resonances of the linear $(m=2)$ and first nonlinear $(m=3)$ modes only [12-19]; it is unnecessary to seek for their origins in much higher-order driving terms. Extra attention to the fourth and high-order nonlinear resonances may, however, be required in a circular machine that stores intense hadron bunches for a very long period (see Appendix C).

The seed of parametric beam resonance is a distortion of the particle distribution from the ideal stationary state finegrainedly matched to the external AG potential in phase space. Since perfect matching can never be achieved in practice, any realistic beam will sooner or later start to blow if the machine operating condition is poorly chosen. At an operating point where coherent resonances of relatively low orders overlap, we sometimes observe the development of a high-order instability first followed by a lower-order's. This does not necessarily mean that the higher-order resonance is stronger than the lower-order resonance or the latter can only be activated through the preceding disturbance to the initial particle distribution by the former instability. Even without a disturbance from the higher-order mode, the lower-order instability will eventually grow resulting in more serious particle losses. The resonance of a lower order is generally more troublesome unless the external driving potential contains excess nonlinearity [25] (or the beam initially possesses an unusual distribution very far from the stationary state). We should also keep it in mind that coherent resonances of different orders have different widths and different space-charge-induced band shifts because of the mode-dependent factor $C_{m}[22,25]$. The phase-space structure of an unstable beam may, therefore, evolve quite differently even at two adjacent operating points within a local instability region (cf. Fig. 3). Furthermore, the effective operating point gradually moves as the phase shift $\Delta \bar{\sigma}$ is reduced by emittance growth. All these complex features of the collective resonance process make it extremely difficult to explain the time evolution of the beam behavior in phase space.

It is apparent from Fig. 10 that at least two low-order resonances excited independently in the horizontal and vertical directions contribute to each of the three pronounced instability bands discovered in Figs. 6-9 for the symmetric beam transport. In the general case where $\sigma_{0 x} \neq \sigma_{0 y}$, we must particularly care about the three vertical lines $\sigma_{0 x} \approx 60^{\circ}, 90^{\circ}$, $120^{\circ}$ and the three horizontal lines $\sigma_{0 y} \approx 60^{\circ}, 90^{\circ}, 120^{\circ}$ on the tune diagram. Among them, the instability bands just above $\sigma_{0 x(0 y)}=90^{\circ}$ are most dangerous whenever the beam is dense $[13,15]$. The effect of nonlinear coupling resonance is weak in S-POD II within $10 \mathrm{~ms}$ despite that the rf field in the LPT aperture certainly involves finite nonlinearities. Assuming the rms misalignments of the LPT electrodes to be a few tens of $\mu \mathrm{m}$, the resultant sextupole and octupole nonlinearities are on the order of $0.1 \%$ relative to the quadrupole strength. This level of nonlinearity is comparable to or, at least, not too far from possible imperfections expected in real machines [55,62-64]. For example, the strengths of low-order nonlinear components are $0.1 \%$ or a 
bit smaller in the quadrupole magnets used for the DTL at J-PARC, where the FODO lattice has been adopted for transverse focusing of high-intensity proton beams $[62,65]$. The actual strengths of nonlinear error fields along the beam line should be increased to some degree because of the limited accuracy of the drift-tube alignments [66]. We thus believe that the present S-POD data have reproduced what is likely to happen in conventional linear machines for hadron beams. Considering the fact that the ion storage period of $10 \mathrm{~ms}$ in the LPT is equivalent to beam transport over 10,000 FODO cells, nonlinear coupling resonances are probably of little concern in practice when the beam is near the ideal stationary state at injection. In a strongly asymmetric lattice, however, it is recommended to care about several coupling resonance lines experimentally revealed at high intensity on the tune diagrams in Figs. 10(c) and 11; namely, the sum resonance $\sigma_{0 x}+\sigma_{0 y} \approx 180^{\circ}$ [59] and third-order difference resonances $2 \sigma_{0 x}-\sigma_{0 y} \approx 0$ and $\sigma_{0 x}-2 \sigma_{0 y} \approx 0$, all of which are observable even in short-term PIC simulations without external nonlinearities (see Appendix B).

The growth rate and stop-band width of self-field-driven resonance are roughly proportional to the beam perveance [25]. The coherent instabilities of higher than the third order $(m \geq 4)$ could, therefore, cause unacceptable emittance growth even in a linear transport channel if the tune depression is significantly below the range considered in the present study. All stop bands observed in the stability map of Fig. 10 then become much wider and, in addition, some more coupling resonance lines may manifest themselves depending on the beam density and transport distance (cf. Appendices B and C). For the future study of such an extremely space-charge-dominant regime with S-POD, we are now developing a plasma stacking technique to accumulate a large number of ${ }^{40} \mathrm{Ca}^{+}$ions in the LPT [41]. The use of ${ }^{40} \mathrm{Ca}^{+}$ions allows the application of powerful Doppler laser cooling [67,68], while the tune depression currently reachable with ${ }^{40} \mathrm{Ar}^{+}$ions is limited to about $0.8-0.85$ due to technical reasons. In principle, a bunch of ${ }^{40} \mathrm{Ca}^{+}$ions can be cooled to near the absolute zero temperature, which means that it is possible to depress the betatron tune down to the ultimate lower limit, i.e., $\eta \approx 0$ where the beam is Coulomb crystallized.

\section{ACKNOWLEDGMENTS}

This work is supported in part by Japan Society for the Promotion of Science (JSPS) KAKENHI Grant Number $15 \mathrm{H} 03662$. The authors are indebted to M. Ota and T. Okano for providing experimental data obtained with S-POD III. They also express their sincere thanks to Drs. Y. Iwata, O. Kamigaito, S. Machida, F. Naito, and K. Okabe for useful information of the linear machines at NIRS, RIKEN, and J-PARC. The main contents of this paper are an extended version of a part of the work presented at HB2014 [12] and IPAC2014 [13].
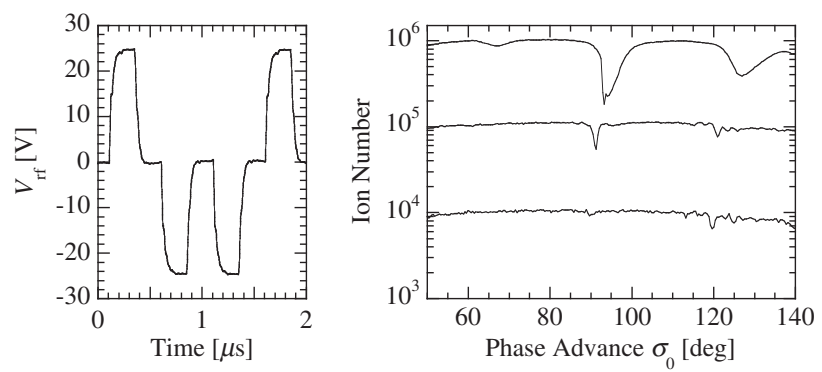

FIG. 12. Ion-loss distributions measured in S-POD with a FDDF focusing potential. The FDDF waveform employed for this experiment is symmetric as depicted in the left panel. The right panel shows the ion-loss distributions at three different initial plasma intensities. The storage period of ${ }^{40} \mathrm{Ar}^{+}$ions in the LPT is fixed at $10 \mathrm{~ms}$ in all measurements. The small ripples in the low-density data are due to the performance limitation of our pulse-waveform generator (rather than to the fluctuation of the initial ion number).

\section{APPENDIX A: FDDF LATTICE}

In addition to the sinusoidal and doublet waveforms considered in Sec. IV, we have also tried other types of AG waveforms for $K_{\mathrm{rf}}(\tau)$ including the so-called "triplet," "FDDF," etc. [12,13]. The left panel in Fig. 12 shows an example of the rf voltage generated by the S-POD power supply to emulate a FDDF-type lattice configuration. Since the lattice is symmetric, the horizontal and vertical phase advances are equal; namely, $\sigma_{0 x}=\sigma_{0 y}\left(\equiv \sigma_{0}\right)$. The rf period is now $2 \mu$ s, twice longer than the doublet case. The plasma envelope is then a bit more expanded in the LPT aperture, which enhances ion losses; in other words, the acceptance of the LPT is reduced to some degree. It is, therefore, difficult to confine as large a number of ions as in the case of the FODO lattice, but the global picture of the stop-band distribution is not substantially changed. When the plasma density is high, the three instability regions are formed just above $\sigma_{0}=60^{\circ}, 90^{\circ}$, and $120^{\circ}$ as expected. The severe $90^{\circ}$ stop band disappears in the emittance-dominated regime while finite ion losses remain at $\sigma_{0} \approx 120^{\circ}$.

\section{APPENDIX B: SELF-FIELD-DRIVEN COUPLING RESONANCES}

It is natural to expect that the Coulomb self-field potential can be a source of not only the parametric 1D resonances in Eq. (5) but also parametric coupling resonances. In the 2D case, however, the fully analytic derivation of a general resonance condition from the Vlasov-Poisson equations is hopeless to achieve. All we can do is to develop a hypothesis based on the 1D criterion and numerical data. A simple-minded generalization of the condition in Eq. (5) is

$$
k\left(\sigma_{0 x}-C_{k \ell}^{(x)} \Delta \bar{\sigma}_{x}\right) \pm \ell\left(\sigma_{0 y}-C_{k \ell}^{(y)} \Delta \bar{\sigma}_{y}\right) \approx n \pi,
$$


where $C_{k \ell}^{(x)}$ and $C_{k \ell}^{(y)}$ are constant parameters depending on the order of the driving space-charge term $x^{k} y^{\ell}$, and $\Delta \bar{\sigma}_{x(y)}$ represent the coherent phase shifts in the two transverse directions. The resonance order is $k+\ell(\equiv m)$. We require $C_{m 0}^{(x)}=C_{0 m}^{(y)}=C_{m}$ so that Eq. (B1) is reduced to Eq. (5) for the 1D coherent instability in the horizontal or vertical degree of freedom. Because of the symmetry of the problem, the requirement $C_{k \ell}^{(x)}=C_{\ell k}^{(y)}$ should also be met. The space-charge-induced phase shifts are defined as $\Delta \bar{\sigma}_{x(y)}=\left(1-\eta_{x(y)}\right) \sigma_{0 x(0 y)}$ with $\eta_{x(y)}$ being the horizontal(vertical) rms tune depression. The condition above has been linearized with respect to the phase shift, assuming $\Delta \bar{\sigma}_{x(y)} / \sigma_{0 x(0 y)} \ll 1$. The parameter dependence could be more complex at very high beam density. The right-hand side of Eq. (B1) must be replaced by $2 n \pi$ if the driving force comes from an artificial source, e.g., mechanical imperfections [12].

We can show the possibility of self-field-driven coupling resonances by generalizing the procedure in Ref. [24], but the Vlasov theory based on the KV model predicts so many nonlinear stop bands (even if we ignore all resonances higher than the fourth order), most of which are probably unimportant in practice [60]. As experimentally demonstrated with S-POD for the first time, the number of observable coupling resonance lines is limited even after 10,000 AG periods in the absence of strong external nonlinearities. Our experimental observations in Sec. IV agree fairly well with PIC simulations in Fig. 13 where the rms emittance growth rate after $100 \mathrm{AG}$ focusing periods is color-coded on the tune diagram. The horizontal and vertical tune depressions are set equal initially to $\eta_{x}=\eta_{y}=0.9$. Since no nonlinear imperfection fields have been taken into account here, the resonances manifested in Fig. 13 are purely space-charge-driven. Apart from the 1D resonance lines confirmed in Fig. 10, we notice five coupling resonance lines expressed approximately as

$$
\begin{gathered}
\sigma_{0 x}\left[1-(1-\eta) C_{11}^{(x)}\right]+\sigma_{0 y}\left[1-(1-\eta) C_{11}^{(y)}\right] \approx \pi, \\
\left\{\begin{array}{l}
2 \sigma_{0 x}\left[1-(1-\eta) C_{21}^{(x)}\right]-\sigma_{0 y}\left[1-(1-\eta) C_{21}^{(y)}\right] \approx 0, \\
\sigma_{0 x}\left[1-(1-\eta) C_{12}^{(x)}\right]-2 \sigma_{0 y}\left[1-(1-\eta) C_{12}^{(y)}\right] \approx 0,
\end{array}\right. \\
\left\{\begin{array}{l}
2 \sigma_{0 x}\left[1-(1-\eta) C_{21}^{(x)}\right]-\sigma_{0 y}\left[1-(1-\eta) C_{21}^{(y)}\right] \approx \pi, \\
\sigma_{0 x}\left[1-(1-\eta) C_{12}^{(x)}\right]-2 \sigma_{0 y}\left[1-(1-\eta) C_{12}^{(y)}\right] \approx-\pi,
\end{array}\right.
\end{gathered}
$$

where the definition $\Delta \bar{\sigma}_{x(y)}=\left(1-\eta_{x(y)}\right) \sigma_{0 x(0 y)}$ has been used together with $\eta_{x}=\eta_{y}(\equiv \eta)$. Fitting these lines to the numerical data enables us to estimate the phase-shift factors as follows: $C_{11}^{(x)}=C_{11}^{(y)} \approx 0.7$ and $C_{12}^{(x)}=C_{21}^{(x)}=C_{12}^{(y)}=$ $C_{21}^{(y)} \approx 0.8$. Interestingly, these numbers are close, respectively, to $C_{2}$ and $C_{3}$ from the 1D Vlasov prediction [22] and actually usable to fit the $1 \mathrm{D}$ resonance lines as well. These

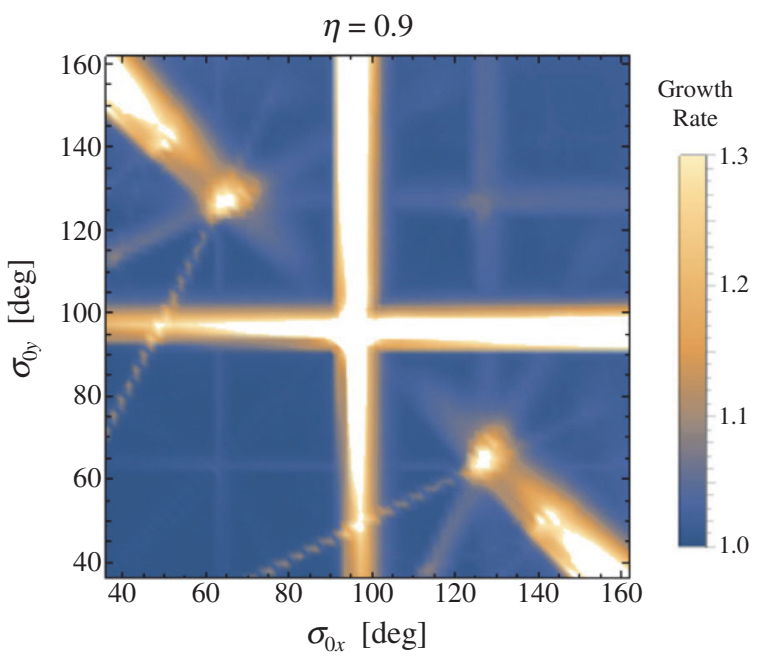

FIG. 13. Tune diagram obtained form 2D WARP simulations at $\eta=0.9$ with no external nonlinearities. The rates of $\mathrm{rms}$ emittance growth numerically evaluated at about 5,000 different operating points are color-coded on the tune diagram. The horizontal and vertical rms emittances are initially unequal almost everywhere on the diagram because the tune depressions in both directions have been adjusted to the same value $\left(\eta_{x}=\eta_{y}=0.9\right)$. When the emittance exchange occurred between the transverse degrees of freedom, we took the larger one of the two growth rates to choose the color. Each simulation is started with a thermally equilibrated beam confined by the sinusoidal focusing potential. The transport channel is 100 sinusoidal periods long. As explained in Sec. II B, the very small emittance growth along the lines $\sigma_{0 x(0 y)} \approx 60^{\circ}$ is most likely due to the sixth-order resonance preceding the sextupole instability. The third-order resonance lines $\sigma_{0 x(0 y)} \approx 120^{\circ}$ are not so clear as in Fig. 10 because of the absence of nonlinear error fields.

findings lead us to a conjecture that the $C$-factor only depends on the order $m$ of the driving potential, namely, $C_{k \ell}^{(x)}=C_{k \ell}^{(y)}=C_{m}$ if the beam is matched to the AG lattice at injection. Equation (B1) can then be simplified to

$$
k \sigma_{0 x} \pm \ell \sigma_{0 y} \approx \frac{n \pi}{1-(1-\eta) C_{m}},
$$

where the positive phase-shift factor $C_{m}(<1)$ approaches unity as the resonance order $m$ becomes higher [22,25]. The two faint difference resonance lines detected experimentally in Fig. 10 correspond to the cases where $(k, \ell, n)=$ $(2,1,0)$ and $(1,2,0)$ in Eq. (B5) with the minus sign. These lines have been reproduced in the PIC simulations in Fig. 13. On the other hand, the third-order difference resonance lines with $(k, \ell, n)=(2,1,1)$ and $(1,2,-1)$ given in Eq. (B4) are invisible in experiment. According to the PIC data, the emittance growth rate along the resonance lines in Eq. (B4) is a few percent or less while the rate is about twice higher along another third-order lines in Eq. (B3). This may be why the weaker resonance lines with $n= \pm 1$ are undetectable with S-POD. 


\section{APPENDIX C: LONG-TERM BEAM STABILITY IN HIGH-INTENSITY HADRON STORAGE RINGS}

In a circular machine, even if it is a so-called highintensity ring, the Coulomb self-field potential is not so strong as achievable in S-POD; the space-charge-induced tune shift is usually below $10 \%$ of the bare tune, i.e., $\eta>0.9$. Nevertheless, we have to be careful about various nonlinear resonance lines because, unlike linear transport channels, the beam often stays in the machine for much longer than 10,000 AG periods assumed in Sec. IV. Note also that the tune depression is determined by the beam density in phase space rather than by the beam intensity or power in real space. Even if the beam is compressed in real space by a conservative electromagnetic force, the phasespace density does not change due to Liouville's theorem. To control $\eta$, the beam must be compressed in phase space The space-charge-driven resonance may thus limit the performance of a cooler storage ring of hadron beams. As a matter of fact, the stop band of the linear $(m=2)$ coherent instability has been known to interrupt the cooling process seriously $[69,70]$. It is one of the most critical limiting factors for next-generation cooler rings aiming at the production of ultralow-emittance ion beams [71].

The resonance lines of the seventh $(m=7)$ and higher than eighth $(m \geq 9)$ orders are hardly visible in S-POD experiments so far even if we extend the plasma storage period to a few hundred milliseconds. On the other hand, it is always easier to spot an instability band near $\sigma_{0}=360^{\circ} / 8$. A typical result on long-term plasma stability at low intensity is plotted in Fig. 14(a) for reference. The ion storage period is now $300 \mathrm{~ms}$ (equivalent to beam transport over $3 \times 10^{5} \mathrm{AG}$ cells), thirty times longer than the experiments in Sec. IV. Noticeable ion losses have occurred at the phase advances close to $\sigma_{0}=360^{\circ} / j$ with $j=3,4,5,6$, and 8 . The space-charge-induced shift of each stop band is small but nonzero. The possible instability at $\sigma_{0}=360^{\circ} / 7$ is missing even in this time scale. It is noteworthy that the instabilities at $\sigma_{0}=360^{\circ} / 6$ and $360^{\circ} / 8$ are stronger than the instability at $\sigma_{0}=360^{\circ} / 5$. These two stop bands become more pronounced as the plasma density is increased. Figure 14(b) shows how the instabilities grow in time at high density. The weak nonlinear resonance of $j=5$ is no longer evident. In addition to the three major resonances observed in Figs. 6-9, one more instability band has been formed at $\sigma_{0} \approx 53^{\circ}$ after $100 \mathrm{~ms}$. This corresponds to the dip originally located at $\sigma_{0}=360^{\circ} / 8$ in Fig. 14(a). Similar tendency has been confirmed also in S-POD III.

According to the interpretation in previous sections, the sextupole resonance $(m=3)$ should be responsible for the instability around $\sigma_{0}=70^{\circ}$, which is why the loss rate there is much higher than that of the fifth-order resonance (expected near $\sigma_{0}=80^{\circ}$ at this intensity). A similar argument can be made regarding the primary source of the instability at $\sigma_{0} \approx 53^{\circ}$ in Fig. 14(b). We presume that this

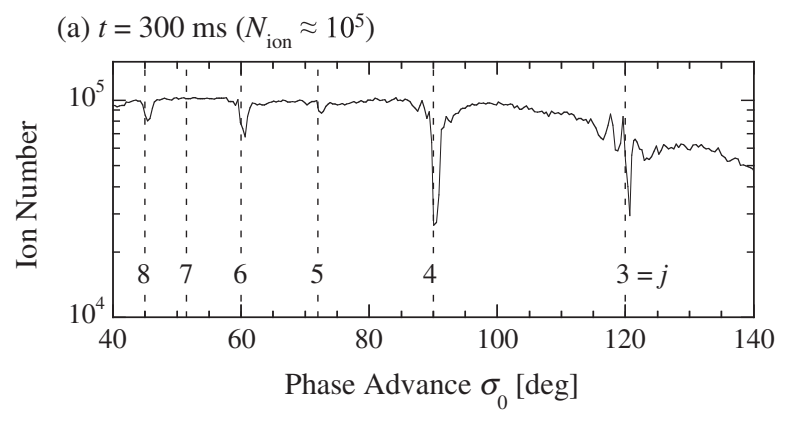

(b) Time evolution

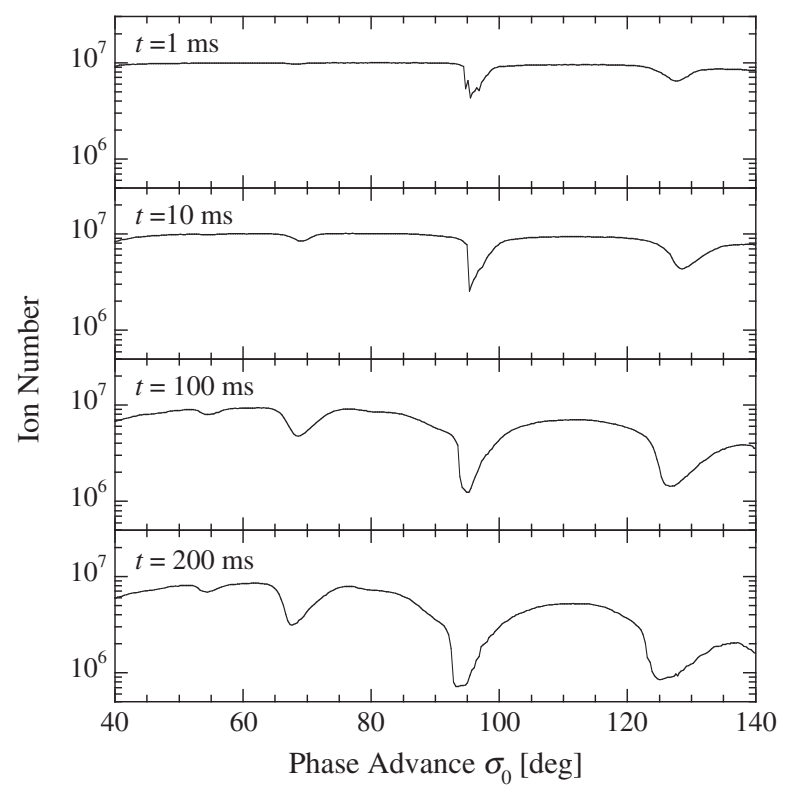

FIG. 14. Long-term stability of a non-neutral plasma confined in S-POD by the sinusoidal focusing potential. (a) Stop-band distribution measured at the initial ion number $N_{\text {ion }} \approx 10^{5}$. The storage period of ions is fixed at $300 \mathrm{~ms}$ corresponding to $3 \times 10^{5}$ AG periods. The vertical broken lines indicate the positions of the phase-advance values $360^{\circ} / j(j=3,4, \ldots, 8)$. (b) Time-evolution of the stop-band distribution in the space-charge-dominant regime where $N_{\text {ion }} \approx 10^{7}$.

instability is driven not by the eighth-order $(m=8)$ imperfection but rather by the much lower fourth-order $(m=4)$ term in the space-charge potential. It is unreasonable to assert that the eighth-order error field has been accidentally created much more strongly than the fifthorder error in both S-POD II and III. All of the prominent instability bands observed in Fig. 14(b) at high density can then be explained as collective resonances lower than the fifth order $(m \leq 4)$. The instabilities near $\sigma_{0}=360^{\circ} / 5$ and $360^{\circ} / 7$ originate purely from the odd-order modes of $m=5$ and $m=7$ higher than the fourth order, so they are weaker.

Suppose a storage ring composed of $N_{\text {sp }}$ lattice superperiods. The coherent resonance conditions in Eqs. (5) and (8) can be put together as 


$$
\nu_{0}-C_{m} \Delta \bar{\nu} \approx \begin{cases}N_{\mathrm{sp}} \cdot \frac{n}{2 m} & (\text { self-field-driven }) \\ N_{\mathrm{sp}} \cdot \frac{n}{m} & (\text { external-field-driven })\end{cases}
$$

where $\nu_{0}$ represents the bare betatron tune around the ring in either the horizontal or vertical direction, and $\Delta \bar{\nu}=$ $(1-\eta) \nu_{0}[12,14,19]$. Let us consider the case of CERN PS as an example. Wasef et al. has reported experimental evidence indicating the excitation of an intensity-dependent resonance at $\nu_{0} \approx 6.25$ [9]. The unit lattice of the PS is the FDDF type studied in Appendix A. This lattice possesses the resonance property analogous to that of the doublet and sinusoidal focusing channels. Since the PS consists of 50 FDDF blocks, the 50th driving harmonic is expected to have a non-negligible amplitude even though the strict superperiodicity of the machine is 10 . If we set $N_{\text {sp }}=50$, the self-field-driven resonance condition in Eq. (C1) predicts the fourth-order (not the eighth-order) instability slightly above $\nu_{0}=6.25$.

If the conjecture made in Appendix B is valid, Eq. (C1) is generalized for an initially matched intense beam as follows:

$$
k \nu_{0 x} \pm \ell \nu_{0 y} \approx \begin{cases}\frac{N_{\mathrm{sp}}}{1-(1-\eta) C_{m}} \cdot \frac{n}{2} & \text { (self-field-driven) } \\ \frac{N_{\mathrm{sp}}}{1-(1-\eta) C_{m}} \cdot n & \text { (external-field-driven) }\end{cases}
$$

where $\left(\nu_{0 x}, \nu_{0 y}\right)$ are the horizontal and vertical bare tunes around the ring, and $m=k+\ell$. This condition is reduced to Eq. (C1) for the horizontal or vertical 1D coherent resonance of the $m$ th order, namely, in the case where $(k, \ell)=(m, 0)$ or $(0, m)$. In the emittance-dominated regime $(\eta \approx 1)$, the band widths and growth rates of the self-field-driven resonances of all orders are negligible, so we only need to care about the external-field-driven instabilities. Equation (C2) then agrees with the wellknown single-particle resonance condition $k \nu_{0 x} \pm \ell \nu_{0 y} \approx$ $N_{\text {sp }} n$ that depends on the strengths of imperfection fields and nonlinear correction magnets.

[1] E. D. Courant and H. S. Snyder, Theory of the alternatinggradient synchrotron, Ann. Phys. (N.Y.) 3, 1 (1958).

[2] N. C. Christofilos, Focusing System for Ions and Electrons, US Patent No. 2736799 (1950).

[3] Handbook of Accelerator Physics and Engineering, edited by A. W. Chao and M. Tigner (World Scientific, Singapore, 1999) and references therein.

[4] M. Reiser, Theory and Design of Charged Particle Beams (John Wiley \& Sons, New York, 2008) and references therein.
[5] See, e.g., Proceedings of 54th ICFA Advanced Beam Dynamics Workshop on High-Intensity, High Brightness and High Power Hadron Beams (HB2014), Michigan, USA (JACoW, Geneva, 2015).

[6] C. K. Allen et al., Beam-Halo Measurements in HighCurrent Proton Beams, Phys. Rev. Lett. 89, 214802 (2002).

[7] L. Groening et al., Experimental Evidence of the Stop Band in the GSI UNILAC, Phys. Rev. Lett. 102, 234801 (2009).

[8] J. G. Wang, D. X. Wang, H. Suk, and M. Reiser, Reflection and Transmission of Space-Charge Waves at Bunched Beam Ends, Phys. Rev. Lett. 74, 3153 (1995).

[9] R. Wasef, G. Arduini, H. Damerau, S. S. Gilardoni, S. Hancock, C. Hernalsteens, A. Huschauer, and F. Schmidt, Space charge effects and limitations in the CERN Proton Synchrotron, in Proceedings of the 4th International Particle Accelerator Conference (IPAC2013), Shanghai, China (JACoW, Geneva, 2013), WEPEA070, p. 2669.

[10] P. G. O'Shea et al., The University Maryland Electron Ring (UMER), Nucl. Instrum. Methods Phys. Res., Sect. A 464, 646 (2001).

[11] S. Bernal, H. Li, T. Godlove, I. Haber, R. A. Kishek, B. Quinn, M. Reiser, M. Walter, Y. Zou, and P. G. O'Shea, Beam experiments in the extreme space-charge limit on the University of Maryland Electron Ring, Phys. Plasmas 11, 2907 (2004).

[12] H. Okamoto, K. Ito, K. Fukushima, and T. Okano, Recent results from the S-POD trap systems on the stability of intense hadron beams, in Proceedings of the 54th ICFA Advanced Beam Dynamics Workshop on High-Intensity, High-Brightness and High- Power Hadron Beams (HB2014), Michigan, USA (JACoW, Geneva, 2015), TUO2LR03 p. 178.

[13] H. Okamoto, K. Fukushima, H. Higaki, D. Ishikawa, K. Ito, T. Iwai, K. Moriya, T. Okano, K. Osaki, and M. Yamaguchi, Beam dynamics studies with non-neutral plasma traps, in Proceedings of the 5th International Particle Accelerator Conference (IPAC2014), Dresden, Germany (JACoW, Geneva, 2014), FRXAA01, p. 4052.

[14] H. Okamoto, M. Endo, K. Fukushima, H. Higaki, K. Ito, K. Moriya, S. Yamaguchi, and S. M. Lund, Experimental simulation of beam propagation over long path lengths using radio-frequency and magnetic traps, Nucl. Instrum. Methods Phys. Res., Sect. A 733, 119 (2014).

[15] K. Fukushima, K. Ito, H. Okamoto, S. Yamaguchi, K. Moriya, H. Higaki, T. Okano, and S. M. Lund, Experimental verification of resonance instability bands in quadrupole doublet focusing channels, Nucl. Instrum. Methods Phys. Res., Sect. A 733, 18 (2014).

[16] H. Takeuchi, K. Fukushima, K. Ito, K. Moriya, H. Okamoto, and H. Sugimoto, Experimental study of resonance crossing with a Paul trap, Phys. Rev. ST Accel. Beams 15, 074201 (2012).

[17] S. Ohtsubo, M. Fujioka, H. Higaki, K. Ito, H. Okamoto, H. Sugimoto, and S. M. Lund, Experimental study of coherent betatron resonances with a Paul trap, Phys. Rev. ST Accel. Beams 13, 044201 (2010).

[18] K. Moriya, K. Fukushima, K. Ito, T. Okano, H. Okamoto, S. L. Sheehy, D. J. Kelliher, S. Machida, and C. R. Prior, Experimental study of integer resonance crossing in a 
nonscaling fixed field alternating gradient accelerator with a Paul ion trap, Phys. Rev. ST Accel. Beams 18, 034001 (2015).

[19] K. Moriya, M. Ota, K. Fukushima, M. Yamaguchi, K. Ito, and H. Okamoto, Double stop-band structure near halfinteger tunes in high-intensity rings, Phys. Rev. Accel. Beams 19, 114201 (2016).

[20] H. Okamoto and H. Tanaka, Proposed experiment for the study of beam halo formation, Nucl. Instrum. Methods Phys. Res., Sect. A 437, 178 (1999).

[21] The Coulomb coupling constant of an ordinary accelerator beam is much smaller than unity, which means that the collision term is negligible. The inverse of the collision frequency of ${ }^{40} \mathrm{Ar}^{+}$ions in the LPT is estimated to be a few tens of ms or longer in the present S-POD experiment. The collision time between ions and neutral gas atoms is also over a few hundred ms even at the vacuum pressure of $10^{-5}$ $\mathrm{Pa}$. The measured $e$-folding lifetime of the plasma confined in the LPT exceeds $10 \mathrm{~s}$ (equivalent to ten million $\mathrm{AG}$ periods) with $10^{6}{ }^{40} \mathrm{Ar}^{+}$ions. These facts indicate that the effect of interparticle collisions does not affect the S-POD data given in the following sections.

[22] F. J. Sacherer, Ph.D Thesis, Lawrence Radiation Laboratory, 1968; Report No. UCRL-18454, 1968.

[23] R. L. Gluckstern, Oscillation modes in two dimensional beams, in Proceedings of the 1970 Linear Accelerator Conf., edited by M. R. Tracy (Fermilab, Batavia, Illinois, 1970), p. 811.

[24] I. Hofmann, L. J. Laslett, L. Smith, and I. Haber, Stability of the Kapchinskij-Vladimirskij (K-V) distribution in long periodic transport systems, Part. Accel. 13, 145 (1983).

[25] H. Okamoto and K. Yokoya, Parametric resonances in intense one-dimensional beams propagating through a periodic focusing channel, Nucl. Instrum. Methods Phys. Res., Sect. A 482, 51 (2002).

[26] R. Baartman, Betatron Resonances with Space Charge, in Proceedings of Space Charge Physics in High Intensity Hadron Rings (Shelter Island, New York, USA, 1998); AIP Conf. Proc. 448, 56 (1998) and references therein.

[27] F. J. Sacherer, RMS envelope equations with space charge, IEEE Trans. Nucl. Sci. 18, 1105 (1971).

[28] J. Struckmeier and M. Reiser, Theoretical studies of envelope oscillations and instabilities of mismatched intense charged-particle beams in periodic focusing channels, Part. Accel. 14, 227 (1984).

[29] C. Chen and R. C. Davidson, Nonlinear Resonances and Chaotic Behavior in a Periodically Focused Intense Charged-Particle Beams, Phys. Rev. Lett. 72, 2195 (1994).

[30] S. M. Lund and B. Bukh, Stability properties of the transverse envelope equations describing intense ion beam transport, Phys. Rev. ST Accel. Beams 7, 024801 (2004).

[31] C. Li and Y. L. Zhao, Envelope instability and the fourth order resonance, Phys. Rev. ST Accel. Beams 17, 124202 (2014).

[32] J. Struckmeier, J. Klabunde, and M. Reiser, On the stability and emittance growth of different particle phase-space distributions in a long magnetic quadrupole channel, Part. Accel. 15, 47 (1984).

[33] The exact resonance condition derived in Ref. [25] is more complex than Eq. (5). Equation (5) is the simplified version that applies to the particularly severe resonance induced solely by the $m$ th-order mode. The beam stability can also be affected by the coupling of two modes of different orders, but such instability is generally weak. The mathematical formulas of the resonance width and growth rate have been given in Ref. [25].

[34] A. V. Fedotov, I. Hofmann, R. L. Gluckstern, and H. Okamoto, Parametric collective resonances and space-charge limit in high-intensity rings, Phys. Rev. ST Accel. Beams 6, 094201 (2003).

[35] Equation (44) in Ref. [25] is equivalent to Eq. (7) in the present paper.

[36] It is unnecessary or even misleading to introduce a singleparticle instability concept or any extra incoherent mechanism for the explanation of resonance overlapping at high intensity. The coherent resonance conditions in Eqs. (5) and (8) suffice for understanding the essential feature of beam instability experimentally and numerically explored in the following sections.

[37] D. P. Grote, A. Friedman, G. Craig, I. Haber, and W. Sharp, Progress toward source-to-target simulation, Nucl. Instrum. Methods Phys. Res., Sect. A 464, 563 (2001).

[38] S. M. Lund, T. Kikuchi, and R. C. Davidson, Generation of initial kinetic distributions for simulation of long-pulse charged particle beams with high space-charge intensity, Phys. Rev. ST Accel. Beams 12, 114801 (2009).

[39] I. Hofmann and O. Boine-Frankenheim, Space-Charge Structural Instabilities and Resonances in High-Intensity Beams, Phys. Rev. Lett. 115, 204802 (2015).

[40] K. Fukushima and H. Okamoto, Design study of a multipole ion trap for beam physics applications, plasma and fusion research, Plasma Fusion Res. 10, 1401081 (2015).

[41] R. Takai, H. Enokizono, K. Ito, Y. Mizuno, K. Okabe, and H. Okamoto, Development of a compact plasma trap for experimental beam physics, Jpn. J. Appl. Phys. 45, 5332 (2006).

[42] H. Okamoto, Y. Wada, and R. Takai, Radio-frequency quadrupole trap as a tool for experimental beam physics, Nucl. Instrum. Methods Phys. Res., Sect. A 485, 244 (2002).

[43] As discussed in Ref. [40], it is possible to control the periodicity and strength of $\Delta U_{\mathrm{rf}}$ independently of $K_{\mathrm{rf}}$ by introducing extra electrodes into the regular LPT.

[44] The rf power source currently used for S-POD can generate a square wave of up to $250 \mathrm{~V}$ in height. This is more than enough to cover the whole tune range in the case of FODO lattice at the repetition frequency of $1 \mathrm{MHz}$. The rise and fall times of each square pulse are roughly $15 \mathrm{~ns}$, so we usually set the pulse width wider than $100 \mathrm{~ns}$. An arbitrary arrangement of focusing and defocusing pulses with different widths is available, but the number of confinable ions will change depending on the corresponding betatron function, in other words, the size of the matched plasma envelope.

[45] S. Sheehy, D. Kelliher, S. Machida, C. Plostinar, and C. Prior, Overview of the design of the IBEX linear Paul trap, in Proceedings of the 7th International Particle Accelerator Conference (IPAC2016), Busan, Korea (JACoW, Geneva, 2016), WEPOY048, p. 3104. 
[46] E. P. Gilson, R. C. Davidson, P. C. Efthimion, and R. Majeski, Paul Trap Simulator Experiment to Model Intense-Beam Propagation in Alternating-Gradient Transport Systems, Phys. Rev. Lett. 92, 155002 (2004).

[47] E. P. Gilson, R. C. Davidson, M. Dorf, P. C. Efthimion, R. Majeski, M. Chung, M. S. Gutierrez, and A. N. Kabcenell, Studies of emittance growth and halo particle production in intense charged particle beams using the Paul Trap Simulator Experiment, Phys. Plasmas 17, 056707 (2010).

[48] E. P. Gilson, R. C. Davidson, P. C. Efthimion, R. Majeski, E. A. Startsev, H. Wang, S. Koppell, and M. Talley, Excitation of transverse dipole and quadrupole modes in a pure ion plasma in a linear Paul trap to study collective processes in intense beams, Phys. Plasmas 20, 055706 (2013).

[49] M. Chung, E. P. Gilson, R. C. Davidson, P. C. Efthimion, and R. Majeski, Use of a Linear Paul Trap to Study Random Noise-Induced Beam Degradation in High-Intensity Accelerators, Phys. Rev. Lett. 102, 145003 (2009).

[50] M. Chung, E. P. Gilson, R. C. Davidson, P. C. Efthimion, and R. Majeski, Experimental investigation of random noise-induced beam degradation in high-intensity accelerators using a linear Paul trap, Phys. Rev. ST Accel. Beams 12, 054203 (2009).

[51] H. Higaki, K. Ito, R. Takai, K. Nakayama, W. Saiki, K. Izawa, and $\mathrm{H}$. Okamoto, Axial resonances of $\mathrm{Ar}^{+}$ions observed in a linear Paul trap, Hyperfine Interact. 174, 77 (2007).

[52] A short bunch can readily be produced by shortening the length of the quadrupole rods in the plasma confinement region.

[53] We have confirmed the dependence of ion losses near $\sigma_{0}=120^{\circ}$ on the degree of mechanical imperfections. See, e.g., Fig. 4 of Ref. [14] in which ion-loss distributions measured in two separate LPTs are plotted. Ion losses near $\sigma_{0}=120^{\circ}$ are much greater in S-POD III (broken line), which suggests that the LPT has larger fabrication errors.

[54] N. Kjærgaard, K. Mølhave, and M. Drewsen, Stability of Coulomb crystals in a linear Paul trap with storage-ringlike confinement, Phys. Rev. E 66, 015401(R) (2002).

[55] T. Wangler, RF Linear Accelerators (John Wiley \& Sons, New York, 1998) and references therein.

[56] L. Groening et al., Benchmarking of measurement and simulation of transverse rms-emittance growth, Phys. Rev. ST Accel. Beams 11, 094201 (2008).

[57] Y. Iwata, O. Kamigaito, and K. Okabe (private communication).

[58] In order to form an anisotropic bunch, we horizontally kick the original equilibrium plasma several times with the electric dipole field. The dipole potential can easily be produced within the LPT aperture; all we need is to apply pulse voltages of opposite signs onto the horizontal electrode pair [19]. The pulse widths, heights, and excitation timings of the dipole kicks are carefully chosen to induce a proper plasma displacement for horizontal scraping by the electrodes. A set of the dipole kicks is also designed such that the plasma centroid comes back to the center of the LPT aperture after the scraping. This procedure is equivalent to inserting a local orbital bump in a beam transport line by bending magnets.

[59] O. Boine-Frankenheim, I. Hofmann, and J. Struckmeier, Parametric sum envelope instability of periodically focused intense beams, Phys. Plasmas 23, 090705 (2016).

[60] Y. Tokashiki, K. Fukushima, and H. Okamoto, Twodimensional Vlasov analysis of collective resonances in intense charged-particle beams, in Proceedings of the 71st Annual Meeting of the Physical Society of Japan, 2016 (The Physical Society of Japan, Tokyo, 2016), 19aAQ-1, p. 553.

[61] Since we have measured not rms emittance but the number of surviving ions with a FC, actual stop bands may be a bit wider than the ion-loss regions confirmed on the tune diagram in Fig. 10.

[62] Accelerator Technical Design Report for High-Intensity Proton Accelerator Facility Project, J-PARC, edited by Y. Yamazaki, Report No. JAERI-Tech 2003-044, KEK Report2002-13, 2003.

[63] L. Arnaudon et al., Linac4 Technical Design Report, edited by F. Gerigk and M. Vretenar, CERN-AB-2006-084 ABP/ RF, 2006.

[64] G. W. Wheeler, K. Batchelor, R. Chasman, P. Grand, and J. Sheehan, The brookhaven 200-mev proton linear accelerator, Part. Accel. 9, 1 (1979).

[65] K. Yoshino et al., Development of a DTL quadrupole magnet with a new electroformed hollow coil for the JAERI/KEK joint project, in Proceedings of $X X$ International Linac Conference (LINAC2000), California, USA (SLAC, California, 2000), TUD10, p. 569.

[66] F. Naito et al., Mechanical and rf properties of the DTL for the JAERI/KEK joint project, in Proceedings of the 21st International Linac Conference (LINAC2002), Gyeongju, South Korea (Pohang Accelerator Laboratory, Pohang, 2002), TU424, p. 359.

[67] D. Wineland and H. Dehmelt, Proposed $10^{14} \nu / \Delta \nu$ laser fluorescence spectroscopy on $\mathrm{Tl}^{+}$mono-ion oscillator III, Bull. Am. Phys. Soc. 20, 637 (1975).

[68] T. W. Hänsch and A. L. Schawlow, Cooling of gases by laser radiation, Opt. Commun. 13, 68 (1975).

[69] N. Madsen, P. Bowe, M. Drewsen, L. H. Hornekær, N. Kjærgaard, A. Labrador, J. S. Nielsen, J. P. Schiffer, P. Shi, and J.S. Hangst, Density Limitation in a Stored Laser-Cooled Ion Beam, Phys. Rev. Lett. 83, 4301 (1999).

[70] K. Okabe and H. Okamoto, Emittance limitation in cooled hadron beams, Jpn. J. Appl. Phys. 42, 4584 (2003).

[71] J. Wei, H. Okamoto, and A. M. Sessler, Necessary Conditions for Attaining a Crystalline Beam, Phys. Rev. Lett. 80, 2606 (1998). 